\title{
Extension of Highly Elliptical Earth Orbits using Continuous Low-Thrust Propulsion
}

\author{
Pamela Anderson ${ }^{*}$ and Malcolm Macdonald ${ }^{\dagger}$ \\ University of Strathclyde, Glasgow, G1 1XJ, Scotland, E.U.
}

The extension of highly elliptical orbits, with free selection of orbit period, using lowthrust propulsion is investigated. These newly proposed orbits, termed Taranis orbits, are enabled by existing low-thrust propulsion technology, offering a radically new set of tools for mission design and facilitating new, novel Earth Observation science. One particular example considered herein, using general and special perturbation techniques, is the application of continuous low-thrust to alter the 'critical inclination' of an orbit from the natural values of $63.4 \mathrm{deg}$ or $116.6 \mathrm{deg}$, to any inclination required to optimally fulfill the mission goals. This continuous acceleration is used to compensate for the drift in argument of perigee caused by Earth's gravitational field. Pseudo-spectral optimization techniques are applied to the 90deg inclination Taranis orbit, generating fuel optimal low-thrust control profiles, with a fuel saving of $\sim 4 \%$ from general perturbation results. This orbit provides an alternative solution for high latitude imaging from distances equivalent to geostationary orbits. Analysis shows that the orbit enables continuous, high elevation visibility of frigid and neighboring temperate regions using only three spacecraft, whereas a Molniya orbit would require in excess of fifteen spacecraft, thus enabling high quality imaging which would otherwise be prohibited using conventional orbits. Order of magnitude mission lifetimes for a range of mass fractions and specific impulses are also determined. Finally, a Strawman mass budget is developed, where the mission lifetimes for spacecraft with initial mass of $1000 \mathrm{~kg}, 1500 \mathrm{~kg}$, and $2500 \mathrm{~kg}$, are found to be limited to 4.3 years, 6 years and 7.4 years respectively.

\footnotetext{
${ }^{*}$ PhD Candidate, Advanced Space Concepts Laboratory, University of Strathclyde.

${ }^{\dagger}$ Associate Director, Advanced Space Concepts Laboratory, University of Strathclyde. malcolm.macdonald.102@strath.ac.uk, Associate Fellow of the AIAA.
} 


\section{Nomenclature}

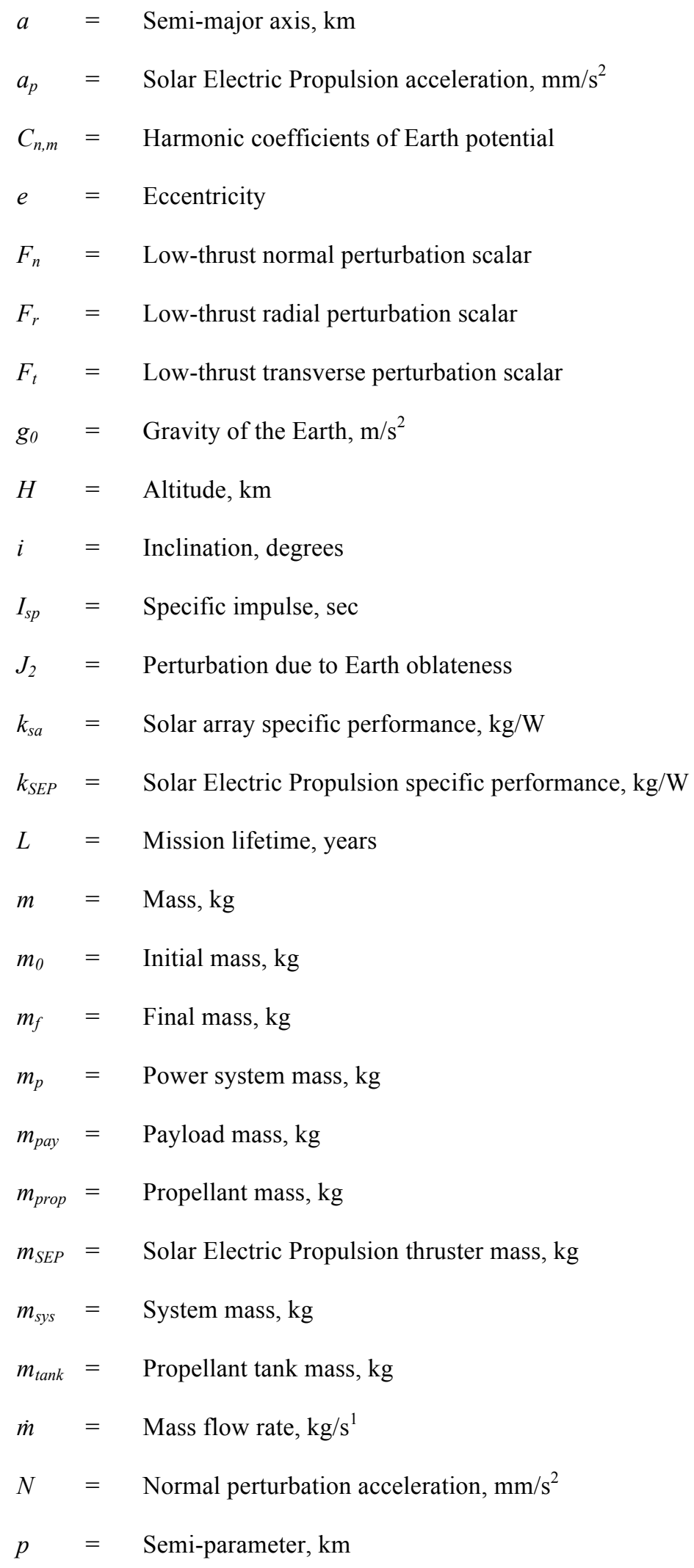




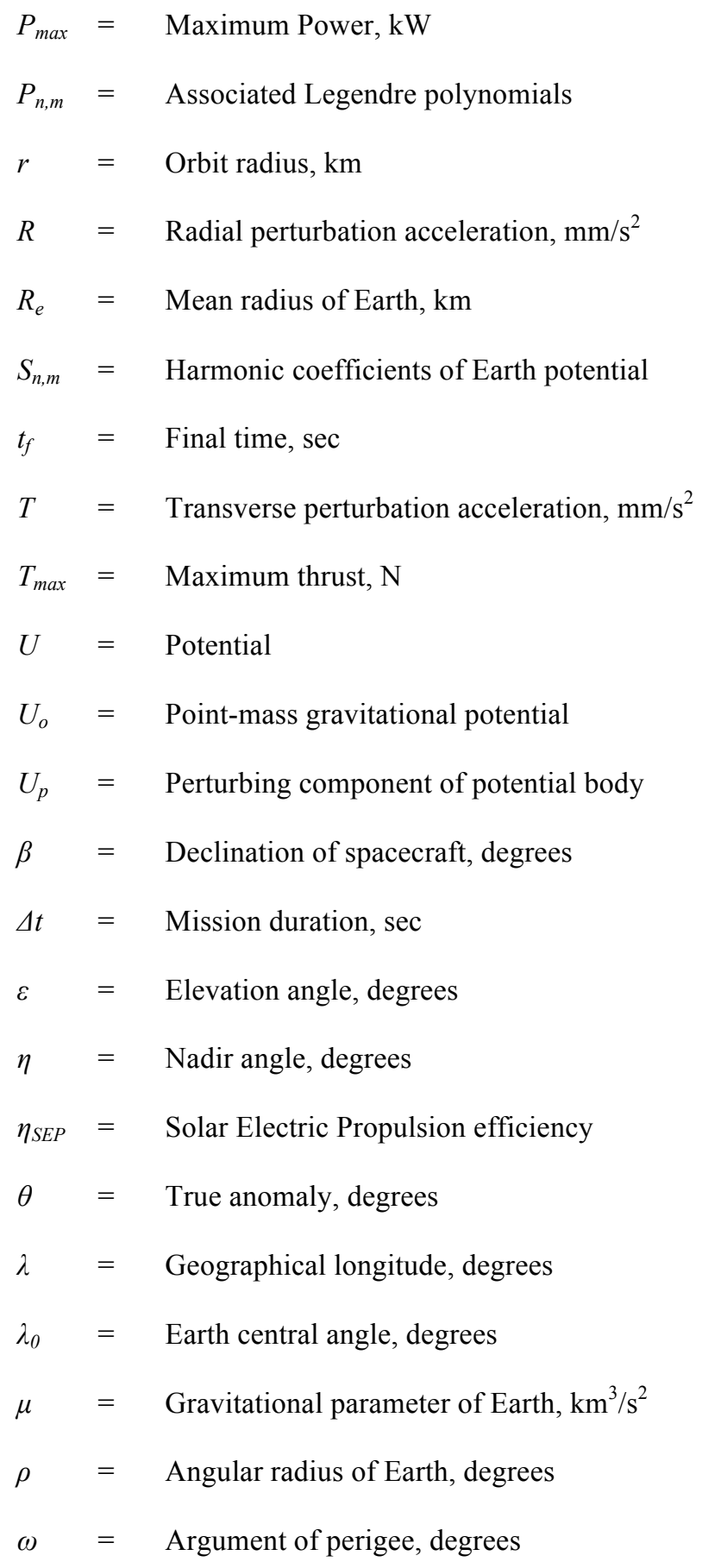

\section{Introduction}

SPACECRAFT provide a unique platform from which to view the Earth and conduct environmental science, offering higher temporal resolution on a global scale than any other observation platform. Consequently, spacebased Earth Observation (EO) measurements are of fundamental importance for validation and assimilation into 
Earth system models. However, the orbits used by spacecraft for remote sensing applications represent only a small subset of those potentially available. It is therefore important to consider whether the currently applied set of orbits can be expanded, introducing new vantage points and enabling novel remote sensing science.

High latitude observations are conventionally conducted using a spacecraft on a Sun-synchronous orbit. With a spacecraft on such an orbit circumnavigating the Earth around fourteen to sixteen times per day [1]. During this time, imaging is conducted by taking measurements over a strip several tens to hundreds of kilometers wide, thus building up an image of a particular location. The problem associated with these orbits is that the temporal resolution provided is insufficient to observe large-scale variations on a diurnal frequency. Temporal resolution is improved by using either multiple spacecraft on similar orbits, or a spacecraft on a GEO. However, to provide continuous observations, with a data refresh rate of less than 15 minutes, as is typically required for so-called continuous meteorological observation, would require a prohibitively large number of spacecraft. Meanwhile, the observational capabilities of geostationary spacecraft are severely limited at latitudes above 55deg [2] due to the rapidly decreasing horizontal resolution and increasingly oblique viewing angle. As such, the breadth and depth of climate and meteorological data which is available for the tropics and mid-latitudes must be approximated by composite or mosaic images beyond around 55deg [2]. However, even with the large number of spacecraft required to provide a data refresh rate of 15 minutes, the data would be discontinuous in time and viewing geometry. Such composites are therefore, at best a compromise, leaving critical climate and meteorological datasets lacking the desired coverage of key frigid and temperate regions required for validation of Earth system models and near realtime monitoring.

The highly elliptical, 12-hr, Molniya orbit overcomes some of the problems associated with imaging of high latitude regions, as a spacecraft on this orbit spends a large amount of time over, say, the Northern Hemisphere as a result of apogee dwell. Characteristic of the Molniya orbits is the fixed 63.4deg or 116.6deg 'critical inclination' [3]. At either of these inclinations there is no rotation of the apsidal line due to the concentration of mass around the Earth's Equator, and the position of apogee remains fixed. However, to ensure at least equal quality imaging to that given by geostationary platforms, an Observational Zenith Angle (OZA) of around 63deg is required to all longitudes at latitudes as far south as 55deg. This OZA cannot be achieved by a conventional Molniya orbit.

A significant amount of work has previously been conducted on the existence, stability and control of displaced Highly Non-Keplerian orbits for the two and three body problem [4-6]. Solar sail, Solar Electric Propulsion (SEP) 
and hybrid solar sail / SEP systems have been considered to enable various missions. For example, recent investigation has been conducted into geostationary orbits (GEO) displaced above or below the equatorial plane to create new geostationary slots to aid the congestion in the conventional GEO ring [7].

Recent research has considered the use of continuous low-thrust propulsion for the extension of Sunsynchronous orbits, enabling free selection of orbit inclination and altitude [8]. Within this recent work, unlike when considering Highly Non-Keplerian Orbits, the thrust magnitude required is not defined as a function of the local gravity field but instead by the magnitude of the perturbations within that field, augmenting the Earth oblateness perturbation to modify the Sun-synchronous orbit.

Hybrid SEP / solar sail systems have recently been proposed to modify the well-known Pole Sitter mission concept, enabling a spacecraft to be statically stationed high above one of the Earth's poles at around 2- 3 million kilometers range. Thus, providing continuous hemispherical observation, throughout the year, using a single spacecraft [9-11]. The Pole Sitter mission offers an alternative to conventional Earth orbits for high latitude imaging; however, to achieve this, a relatively high thrust magnitude of around $200 \mathrm{mN}$ is required [12].

This paper builds on the principle of using low-thrust propulsion to modify the orbit perturbation introduced in [8] and, for the first time, considers the extension of highly elliptical orbits (HEOs), with particular focus on the Molniya orbit. Consideration is given to the application of using continuous low-thrust to modify the critical inclination, while maintaining the zero change in argument of perigee condition essential to a Molniya-like orbit. Noting that Molniya translates from Russian to mean lightning, such low-thrust extensions of the Molniya orbit are herein termed Taranis orbits, after the Celtic god of thunder.

The extension of HEOs using general and special perturbation techniques is presented. Optimization of these results, using pseudo-spectral optimization methods, is conducted for a 12-hr orbit inclined at 90deg. Optimization removes the assumptions which are used to generate the initial analytical expressions and allow a fuel optimal solution to be determined. Mission lifetime analysis and a Strawman mass budget analysis are also presented for a spacecraft on the 12-hr Taranis orbit. In addition to this, a visibility analysis is conducted to derive the number of spacecraft required for both the Molniya and 90deg inclination Taranis orbit to give continuous observation above 55deg latitude, where conventional Earth orbits suffer limitations. The technology requirements to enable these Taranis orbits are also considered, in particular the possibilities for low-thrust ion engines capable of providing the required thrust, as well as sizing of solar arrays and propellant tanks. 


\section{Spacecraft Motion about an Oblate Body}

Taranis orbits effectively use low-thrust propulsion to compensate for the drift in argument of perigee caused by the Earth's gravitational field. Thus, to investigate the effects of low-thrust propulsion applied to HEOs, the gravitational potential of a body is considered [13]

$U(r, \beta, \lambda)=\frac{\mu}{r} \sum_{n=0}^{\infty} \sum_{m=0}^{\infty}\left(\frac{R_{e}}{r}\right)^{n}\left(C_{n, m} \cos (m \lambda)+S_{n, m} \sin (m \lambda)\right) P_{n, m} \sin \beta$

For a body possessing axial symmetry the influence of periodic effects (tesseral and sectorial harmonics) can be neglected for most orbits, and with the notable exception of geostationary orbits this holds true for Earth. The gravitational potential may thus be written as,

$$
U(r, \beta)=\frac{\mu}{r}\left[1-\sum_{n=0}^{\infty} J_{n}\left(\frac{R_{e}}{r}\right)^{n} P_{n} \sin \beta\right]
$$

Expanding Eq. (2), the gravitational potential becomes,

$$
\begin{aligned}
U(r, \beta)= & \frac{\mu}{r}\left[1-J_{2} \frac{1}{2}\left(\frac{R_{e}}{r}\right)^{2}\left(3 \sin ^{2}(\beta)-1\right)\right. \\
& -J_{3} \frac{1}{2}\left(\frac{R_{e}}{r}\right)^{3}\left(5 \sin ^{2}(\beta)-3\right) \sin \beta \\
& -J_{4} \frac{1}{8}\left(\frac{R_{e}}{r}\right)^{4}\left(3-30 \sin ^{2}(\beta)+35 \sin ^{4}(\beta)\right) \\
& -\ldots .]
\end{aligned}
$$

Considering only first order perturbations and using spherical triangle laws Eq. (3) simplifies to,

$$
U(r, \beta)=U_{o}+U_{p}=\frac{\mu}{r}-J_{2} \frac{\mu R_{e}^{2}}{2 r^{3}}\left(3 \sin ^{2}(i) \sin ^{2}(\theta+\omega)-1\right)
$$

The argument of perigee must remain unchanged in order to ensure that the position of apogee is not severely affected by the perturbations caused by the oblate nature of the Earth. Using the Gauss form of the Lagrange 
Planetary Equations, in terms of a spacecraft centered Radial, Transverse, Normal (RTN) coordinate system, the rate of change of argument of perigee is written as [14],

$$
\frac{d \omega}{d \theta}=\frac{r^{2}}{\mu e}\left[-R \cos \theta+T\left(1+\frac{r}{p}\right) \sin \theta\right]-\frac{r^{3}}{\mu p \tan i} \sin (\theta+\omega) N
$$

The disturbing force components due to $J_{2}$ are [15],

$$
\begin{aligned}
& R_{J_{2}}=\frac{3}{2} \frac{J_{2} \mu R_{e}^{2}}{r^{4}}\left(3 \sin ^{2}(i) \sin ^{2}(\theta+\omega)-1\right) \\
& T_{J_{2}}=-\frac{3}{2} \frac{J_{2} \mu R_{e}^{2}}{r^{4}} \sin ^{2}(i) \sin 2(\theta+\omega) \\
& N_{J_{2}}=-\frac{3}{2} \frac{J_{2} \mu R_{e}^{2}}{r^{4}} \sin (2 i) \sin (\theta+\omega)
\end{aligned}
$$

Substituting Eqs. (6) - (8) into Eq. (5) and integrating over one orbital revolution results in the well-known expression for the change in argument of perigee,

$$
(\Delta \omega)_{0}^{2 \pi}=\frac{3 J_{2} \pi R_{e}^{2}(3+5 \cos (2 i))}{4 a^{2}\left(-1+e^{2}\right)^{2}}
$$

To determine the critical inclination, Eq. (9) is set to zero and solved resulting in inclinations of 63.4deg and 116.6deg. Thus, all Earth orbits inclined at these values show no rotation of the apsidal line, irrespective of the values of semi-major axis or eccentricity. These fixed critical inclinations place restrictions on orbit design and thus limit the possible applications of Molniya-like orbits. It is evident from Eq. (9) that altering the inclination from these critical values will result in a drift in the argument of perigee due to the effect of $\mathrm{J}_{2}$ perturbations. Thus, for each value of inclination there exists a constant acceleration magnitude which will negate this drift, and allow free selection of inclination.

\section{Spacecraft Motion about an Oblate Body with Low-Thrust Propulsion}

\section{General Perturbations Technique}


Low-thrust terms are added to the disturbing force components, using the argument of perigee locally optimal control law derived from the variational equation, given in Eq. (5), by consideration of the sine and cosine terms in this equation. Locally optimal control laws maximize the instantaneous rate of change of the argument of perigee, and provide the thrust orientation in analytical form [16]. The locally optimal control law gives the distinct position of the orbit where the sign of the thrust is required to switch direction. The combined $J_{2}$ and low-thrust perturbations in each of the radial, transverse and out-of-plane directions are thus given by,

$$
\begin{aligned}
& R_{J_{2}+F_{r}}=\frac{3}{2} \frac{J_{2} \mu R_{e}^{2}}{r^{4}}\left(3 \sin ^{2}(i) \sin ^{2}(\theta+\omega)-1\right)+F_{r} \operatorname{sgn}[\cos \theta] \\
& T_{J_{2}+F_{t}}=-\frac{3}{2} \frac{J_{2} \mu R_{e}^{2}}{r^{4}} \sin ^{2}(i) \sin 2(\theta+\omega)+F_{t} \operatorname{sgn}[\sin \theta] \\
& N_{J_{2}+F_{n}}=-\frac{3}{2} \frac{J_{2} \mu R_{e}^{2}}{r^{4}} \sin 2(i) \sin (\theta+\omega)+F_{n} \operatorname{sgn}[\sin (\theta+\omega)]
\end{aligned}
$$

where the orbital radius is defined as,

$$
r=\frac{p}{1+e \cos \theta}
$$

The expression for the rate of change of argument of perigee with the application of low-thrust propulsion is determined by inserting Eqs. (10) - (12) into Eq. (5) to give,

$$
\begin{aligned}
\frac{d \omega}{d \theta}= & \left.-\frac{a^{2}\left(1-e^{2}\right)^{2} \cot i \sin (\theta+\omega)\left(F_{n} \operatorname{sgn}[\sin (\theta+\omega)]-\frac{3 J_{2} R_{e}^{2} \mu(1+e \cos \theta)^{4} \sin 2(i) \sin (\theta+\omega)}{2 a^{4}\left(1-e^{2}\right)^{4}}\right)}{\mu(1+e \cos \theta)^{3}}\right) \\
& +\frac{1}{e \mu(1+e \cos \theta)^{2}} a^{2}\left(1-e^{2}\right)^{2}\left(\cos \theta\left(-F_{r} \operatorname{sgn}[\cos \theta]-\frac{3 J_{2} R_{e}^{2} \mu(1+e \cos \theta)^{4}\left(-1+3 \sin ^{2}(i) \sin ^{2}(\theta+\omega)\right)}{2 a^{4}\left(1-e^{2}\right)^{4}}\right)\right) \\
& +\left(1+\frac{1}{1+e \cos \theta}\right) \sin \theta\left(F_{t} \operatorname{sgn}[\sin \theta]-\frac{3 J_{2} R_{e}^{2} \mu(1+e \cos (\theta))^{4} \sin ^{2}(i) \sin 2(\theta+\omega)}{2 a^{4}\left(1-e^{2}\right)^{4}}\right)
\end{aligned}
$$

The change in argument of perigee is found by integrating Eq. (14) over one orbital revolution, employing the assumption that the eccentricity is not equal to zero or one and recognizing the positions on the orbit where the low- 
thrust terms change sign. The total change in argument of perigee is made up of four terms, consisting of the change in argument of perigee due to $J_{2}$ effects and due to the effects of each of the $\mathrm{R}, \mathrm{T}$ and $\mathrm{N}$ accelerations. The total variation in argument of perigee is given by,

$$
(\Delta \omega)_{0}^{2 \pi}=(\Delta \omega)_{J_{2}}+(\Delta \omega)_{F_{r}}+(\Delta \omega)_{F_{t}}+(\Delta \omega)_{F_{n}}
$$

where, each acceleration component is given by,

$$
\begin{aligned}
(\Delta \omega)_{J_{2}}= & \frac{3 J_{2} \pi R_{e}^{2}(3+5 \cos 2(i))}{4 a^{2}\left(-1+e^{2}\right)} \\
(\Delta \omega)_{F_{r}}= & \frac{1}{e \mu} 2 a^{2} F_{r}\left(-2+2 e^{2}-4 e \sqrt{-1+e^{2}} \operatorname{Arctanh}\left[\frac{-1+e}{\sqrt{-1+e^{2}}}\right]\right. \\
& \left.-e \sqrt{-1+e^{2}} \ln \left[\frac{1-e}{\sqrt{-1+e^{2}}}\right]+e \sqrt{-1+e^{2}} \ln \left[\frac{-1+e}{\sqrt{-1+e^{2}}}\right]\right) \\
(\Delta \omega)_{F_{t}} & =-\frac{e \mu}{4 a^{2}\left(-2+e^{2}\right) F_{t}}
\end{aligned}
$$

It is also noted from Eq. (12) that the normal low-thrust component, unlike the radial and transverse low-thrust terms, switches sign as a function of argument of latitude. Consequently, the value assigned to the argument of perigee becomes important in this case. Thus, considering argument of perigee equal to both 0deg and 90deg results in two normal acceleration components, given as,

$$
\begin{aligned}
(\Delta \omega)_{F_{n}}^{\omega=0} & =-\frac{4 a^{2} F_{n} \cos \omega \cot i}{\mu} \\
(\Delta \omega)_{F_{n}}^{\omega=\frac{\pi}{2}} & =\frac{1}{\sqrt{-1+e^{2}} \mu} a^{2} F_{n} \cot i\left(-4 \sqrt{-1+e^{2}}-2 e^{2} \sqrt{-1+e^{2}}+12 e \operatorname{Arctanh}\left[\frac{-1+e}{\sqrt{-1+e^{2}}}\right]\right. \\
& \left.+3 e \ln \left[\frac{1-e}{\sqrt{-1+e^{2}}}\right]-3 e \ln \left[\frac{-1+e}{\sqrt{-1+e^{2}}}\right]\right) \sin \omega
\end{aligned}
$$


Substituting, Eq. (16) - Eq. (20) into Eq. (15) results in two solutions for the change in argument of perigee due to the applied acceleration. It is noted that if no out-of-plane acceleration is considered the two solutions are equal. Initially, consideration is only given to the application of continuous low-thrust in each of the RTN directions individually before multiple acceleration directions are studied. Considering each direction in turn, the analytical expressions, from Eq.(15), are solved using the values of orbital parameters in Table 1 to give the constant acceleration magnitude required to compensate for the effects of the $J_{2}$ perturbation for a range of inclinations, out with the equatorial plane, between $5 \mathrm{deg}$ and $175 \mathrm{deg}$. This is illustrated in Fig. 1.

Table 1 Orbital parameters.

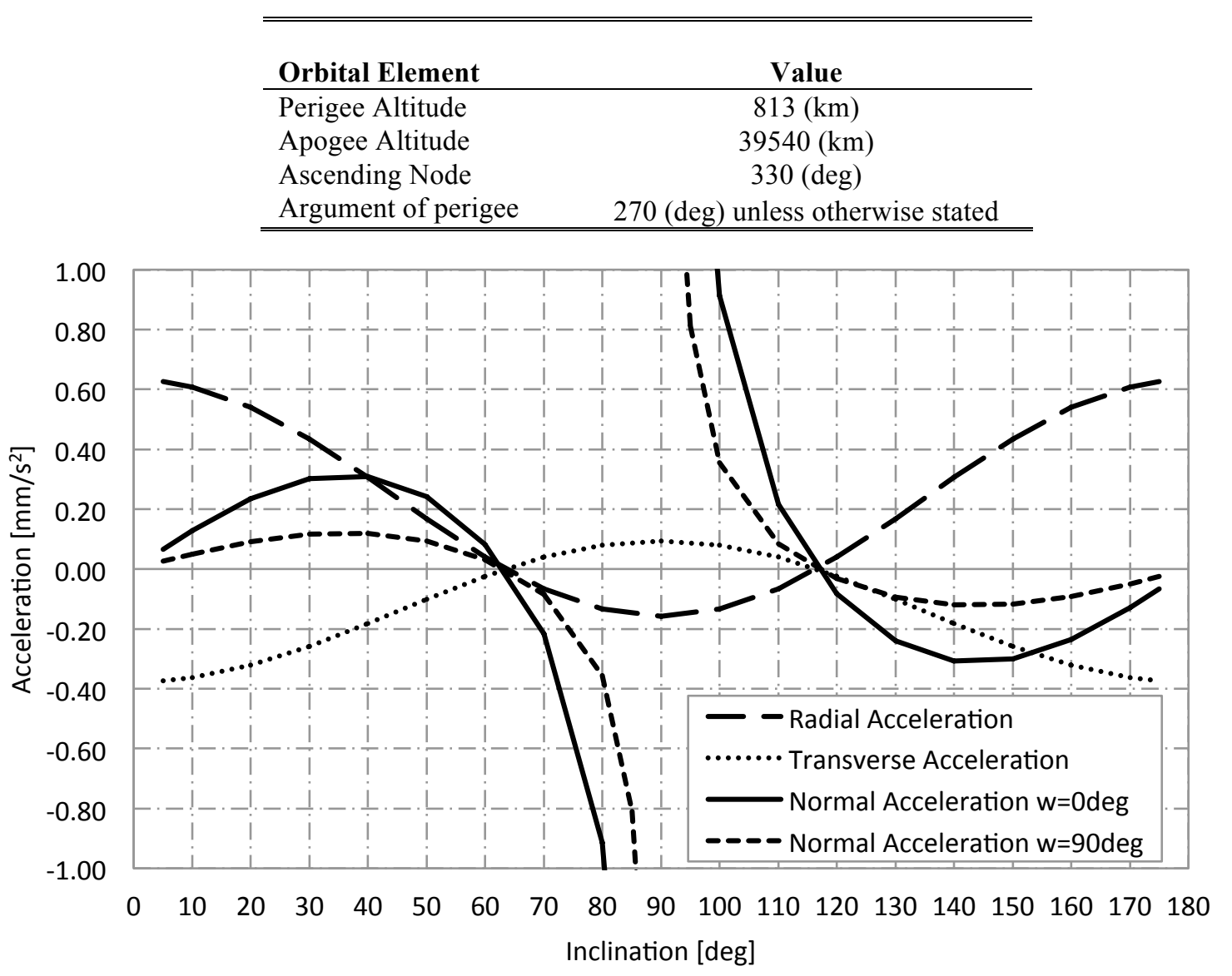

Fig. 1 Required acceleration for thrust along a single axis to vary the critical inclination of the given Taranis orbit.

Figure 1 shows the curves of minimum acceleration in any single axis required to alter the critical inclination of the orbit to a wide range of possible values for the orbit detailed in Table 1. It is noted, from Fig. 1, that a singularity occurs at an inclination of $90 \mathrm{deg}$ when thrusting in the normal direction. This is explained by examining Eqs. (19) (20), where it is shown that normal low-thrust terms in these expressions contain the term cot(i), which at an 
inclination of 90deg becomes undefined causing the singularity. This Figure also illustrates that to reach an inclination of $90 \mathrm{deg}$ by thrusting in any single direction a transverse acceleration of $0.094 \mathrm{~mm} / \mathrm{s}^{2}$ is the lowest acceleration magnitude required. Nevertheless, as shown in Fig. 2 this total magnitude is reduced to $0.0835 \mathrm{~mm} / \mathrm{s}^{2}$ by combining equal magnitudes of radial and transverse accelerations. However, it is of note that the same cannot be said for combining the out-of-plane acceleration with either the radial or transverse accelerations. The cause of this is once again the occurrence of $\cot (i)$ in the Normal direction low-thrust expressions in Eqs. (19) - (20), and the assumption of equal magnitudes in each thrusting direction; as a result of the $\cot (i)$ term the magnitude of this term depends on the inclination to be achieved pushing up the total thrust magnitude. The combined multiple direction, equal magnitude thrust is thus sometimes greater than individual direction thrust magnitude, depending on the value of inclination. This is a clear example that the general perturbation solutions are not optimal.

Although, Fig. 1, shows the results for single axis acceleration for a particular orbit (as detailed in Table 1), the technique used to apply low-thrust to alter the critical inclination and generate the 12-hr Taranis orbit can be used on an orbit of any period. Applying the acceleration in each individual direction to orbits of various periods presents the same trends as shown in Fig. 1, with the acceleration required in each direction decreasing as the orbit period increases. Considering the case where the total acceleration magnitude, required to negate the effect of the $\mathrm{J}_{2}$ perturbation when varying the inclination, is divided equally between radial and transverse directions. Fig. 2 gives the total acceleration required to achieve various inclinations for orbits with periods between 6 and 24 hours, while maintaining the perigee altitude defined in Table 1. 


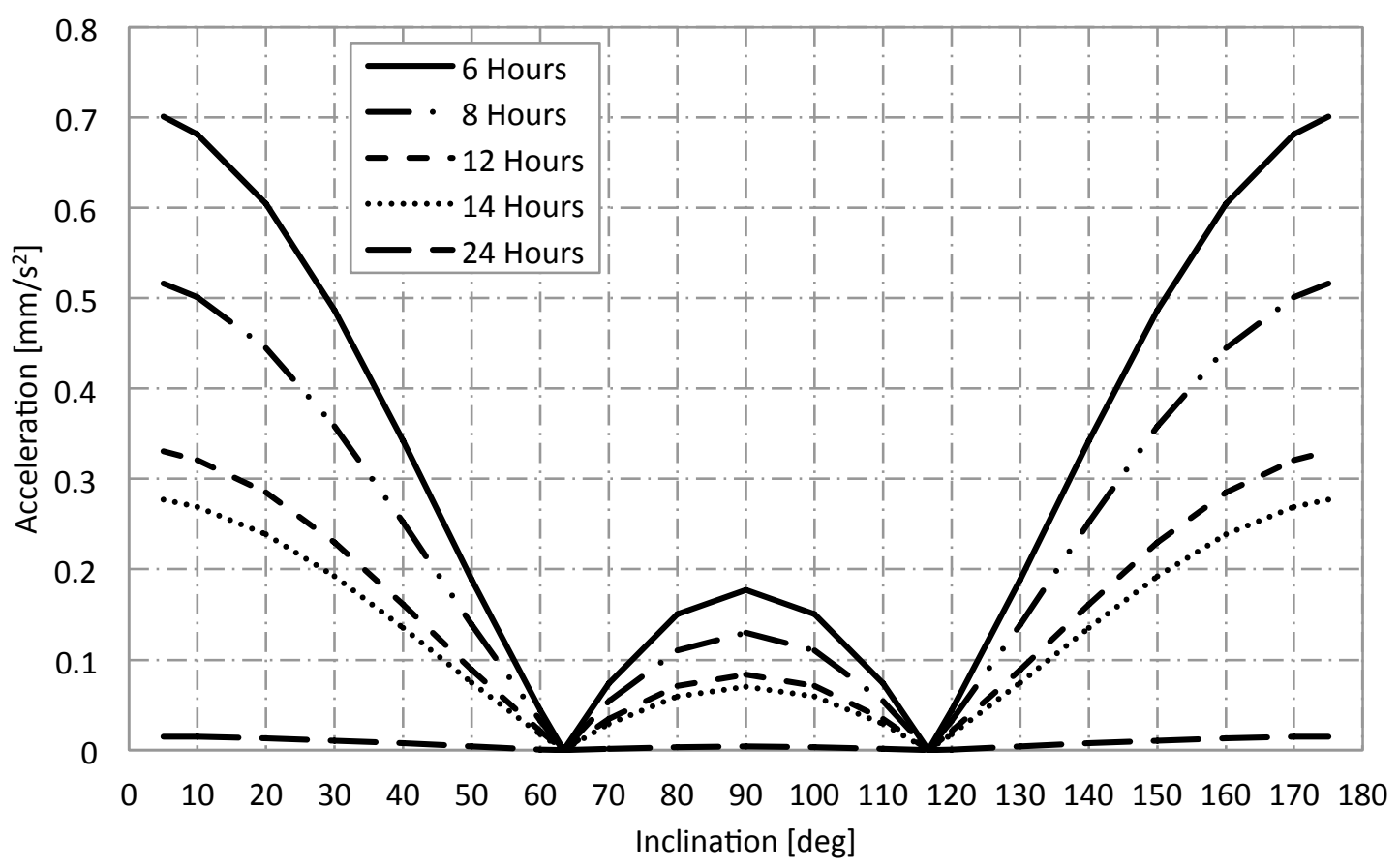

Fig. 2 Combined radial and transverse thrust.

It is seen in Fig. 2 that orbits with lower orbital periods require larger total accelerations than higher period orbits. For example to reach an inclination of $90 \mathrm{deg}$ a 24 hour period requires $0.00378 \mathrm{~mm} / \mathrm{s}^{2}$ acceleration whereas an orbit with a period of 6 hours requires a total acceleration of $0.177 \mathrm{~mm} / \mathrm{s}^{2}$.

\section{Change in Orbital Elements}

Analytical expressions are also developed for the remaining orbital elements, semi-major axis, eccentricity, inclination, and ascending node angle using the Gauss form of the Lagrange Planetary Equations, in terms of a spacecraft centered RTN coordinate system [14]. This process is carried out to ensure the desired zero secular rate of change of orbital elements is maintained in the presence of continuous low-thrust.

1. Semi-major axis

$$
\frac{d a}{d \theta}=\frac{2 p r^{2}}{\mu\left(1-e^{2}\right)^{2}}\left(R_{J_{2}+F_{r}} e \sin \theta+T_{J_{2}+F_{t}} \frac{p}{r}\right)
$$

Substituting appropriate expressions for perturbing forces gives, 


$$
\begin{aligned}
\frac{d a}{d \theta} & =\frac{1}{\mu(1+e \cos \theta)^{2}} 2 a^{3}\left(1-e^{2}\right)\left(e \sin \theta\left(F_{r}+\frac{3 J_{2} R_{e}^{2} \mu(1+e \cos \theta)^{4}\left(-1+3 \sin ^{2}(i) \sin ^{2}(\theta+\omega)\right)}{2 a^{4}\left(1-e^{2}\right)^{4}}\right)\right) \\
& +(1+e \cos \theta)\left(F_{t}-\frac{3 J_{2} R_{e}^{2} \mu(1+e \cos \theta)^{4} \sin ^{2}(i) \sin 2(\theta+\omega)}{2 a^{4}\left(1-e^{2}\right)^{4}}\right)
\end{aligned}
$$

Eq. (22) is then integrated over one orbital revolution to find the change in semi-major axis to give,

$$
(\Delta a)_{0}^{2 \pi}=0
$$

2. Eccentricity

$$
\frac{d e}{d \theta}=\frac{r^{2}}{\mu p}\left(R_{J_{2}+F_{r}} \sin \theta+T_{J_{2}+F_{r}}\left(\left(1+\frac{r}{p}\right) \cos \theta+\frac{r e}{p}\right)\right)
$$

Inserting radial and transverse perturbations into Eq. (24) gives,

$$
\begin{aligned}
\frac{d e}{d \theta} & =\frac{1}{\mu(1+e \cos \theta)^{2}} a\left(1-e^{2}\right)\left(\sin \theta\left(F_{r}+\frac{3 J_{2} R_{e}^{2} \mu(1+e \cos \theta)^{4}\left(-1+3 \sin ^{2}(i) \sin ^{2}(\theta+\omega)\right)}{2 a^{4}\left(1-e^{2}\right)^{4}}\right)\right) \\
& +\left(\frac{e}{1+e \cos \theta}+\cos \theta\left(1+\frac{1}{1+e \cos \theta}\right)\right)\left(F_{t}-\frac{3 J_{2} R_{e}^{2} \mu(1+e \cos \theta)^{4} \sin (i)^{2} \sin 2(\theta+\omega)}{2 a^{4}\left(1-e^{2}\right)^{4}}\right)
\end{aligned}
$$

Integrating over one orbit, the change in eccentricity is written as,

$$
(\Delta e)_{0}^{2 \pi}=0
$$

\section{Inclination}

$$
\frac{d i}{d \theta}=\frac{r^{3}}{\mu p} \cos (\theta+\omega) N_{J_{2}+F_{n}}
$$

Substituting the out-of-plane acceleration, Eq. (26) becomes, 


$$
\frac{d i}{d \theta}=\frac{a^{2}\left(1-e^{2}\right)^{2} \cos (\theta+\omega)}{\mu(1+e \cos \theta)^{3}}\left(F_{n}-\frac{3 J_{2} R_{e}^{2} \mu(1+e \cos \theta)^{4} \sin 2(i) \sin (\theta+\omega)}{2 a^{4}\left(1-e^{2}\right)^{4}}\right)
$$

Once more, the locally optimal control law state that normal thrust switches sign depending on the argument of latitude; consequently Eq. (28) integrated over one revolution using both argument of perigee equal to 0deg and 90deg gives the changes in inclination respectively as,

$$
\begin{aligned}
(\Delta i)_{0}^{2 \pi}= & -\frac{4 a^{2} F_{n} \sin \omega}{\mu} \\
(\Delta i)_{0}^{2 \pi}= & \frac{1}{\sqrt{-1+e^{2}} \mu} a^{2} F_{n} \cos \omega\left(4 \sqrt{-1+e^{2}}+2 e^{2} \sqrt{-1+e^{2}}\right. \\
& \left.-12 e \operatorname{Arctanh}\left[\frac{-1+e}{\sqrt{-1+e^{2}}}\right]-3 e \ln \left[\frac{1-e}{\sqrt{-1+e^{2}}}\right]+3 e \ln \left[\frac{-1+e}{\sqrt{-1+e^{2}}}\right]\right)
\end{aligned}
$$

Inserting values of orbital elements gives the change in inclination for both cases as,

$$
(\Delta i)_{0}^{2 \pi}=0
$$

4. Ascending node

$$
\frac{d \Omega}{d \theta}=\frac{r^{3}}{\mu p \operatorname{sini}} \sin (\theta+\omega) N_{J_{2}+F_{n}}
$$

Inserting the expression for the normal perturbation results in,

$$
\frac{d \Omega}{d \theta}=\frac{a^{2}\left(1-e^{2}\right)^{2} \sin (\theta+\omega)}{\sin i(1+e \cos \theta)^{3}}\left(F_{n}-\frac{3 J_{2} R_{e}^{2} \mu(1+e \cos \theta)^{4} \sin 2(i) \sin (\theta+\omega)}{2 a^{4}\left(1-e^{2}\right)^{4}}\right)
$$

Similar to the inclination, the change in ascending node must be considered using both $\omega=0$ deg and $\omega=90 \operatorname{deg}$ giving the change in ascending node angle for each case respectively as, 


$$
\begin{aligned}
(\Delta \Omega)_{0}^{2 \pi}= & \frac{4 a^{2} F_{n} \cos \omega}{\mu \sin i}-\frac{3 J_{2} \pi R_{e}^{2} \cos i}{a^{2}\left(-1+e^{2}\right)^{2}} \\
(\Delta \Omega)_{0}^{2 \pi}= & -\frac{1}{2 a^{2}\left(-1+e^{2}\right)^{5 / 2} \mu \sin i}\left(3 \sqrt{-1+e^{2}} J_{2} \pi R_{e}^{2} \mu \sin 2(i)+\right. \\
& \left.24 a^{4} e\left(-1+e^{2}\right)^{2} F_{n} \operatorname{Arctanh}\left[\frac{-1+e}{\sqrt{-1+e^{2}}}\right] \sin \omega-2 a^{4}\left(-1+e^{2}\right)^{2} F_{n}\right) \\
& \left(2 \sqrt{-1+e^{2}}\left(2+e^{2}\right)-3 e \ln \left[\frac{1-e}{\sqrt{-1+e^{2}}}\right]+3 e \ln \left[\frac{-1+e}{\sqrt{-1+e^{2}}}\right]\right) \sin \omega
\end{aligned}
$$

Note that if no out-of-plane acceleration is considered; the change in ascending node due only to $J_{2}$ effects is given by,

$$
(\Delta \Omega)_{0}^{2 \pi}=-\frac{3 J_{2} \pi R_{e}^{2} \cos i}{a^{2}\left(-1+e^{2}\right)^{2}}
$$

Orbital element values are substituted into Eqs. (34) - (35) to quantify the drift in ascending node for HEOs, of varying inclinations, over the orbit due to applied normal acceleration. It is of note that when the appropriate orbital elements are substituted into Eqs. (34) - (35), the change in ascending node is equal for both of these expressions. Taranis orbits enabled using continuous normal acceleration are compared with orbits of various inclinations under the influence of $J_{2}$ effects only (from Eq. (36)) the results of which are shown in Fig. 3. 


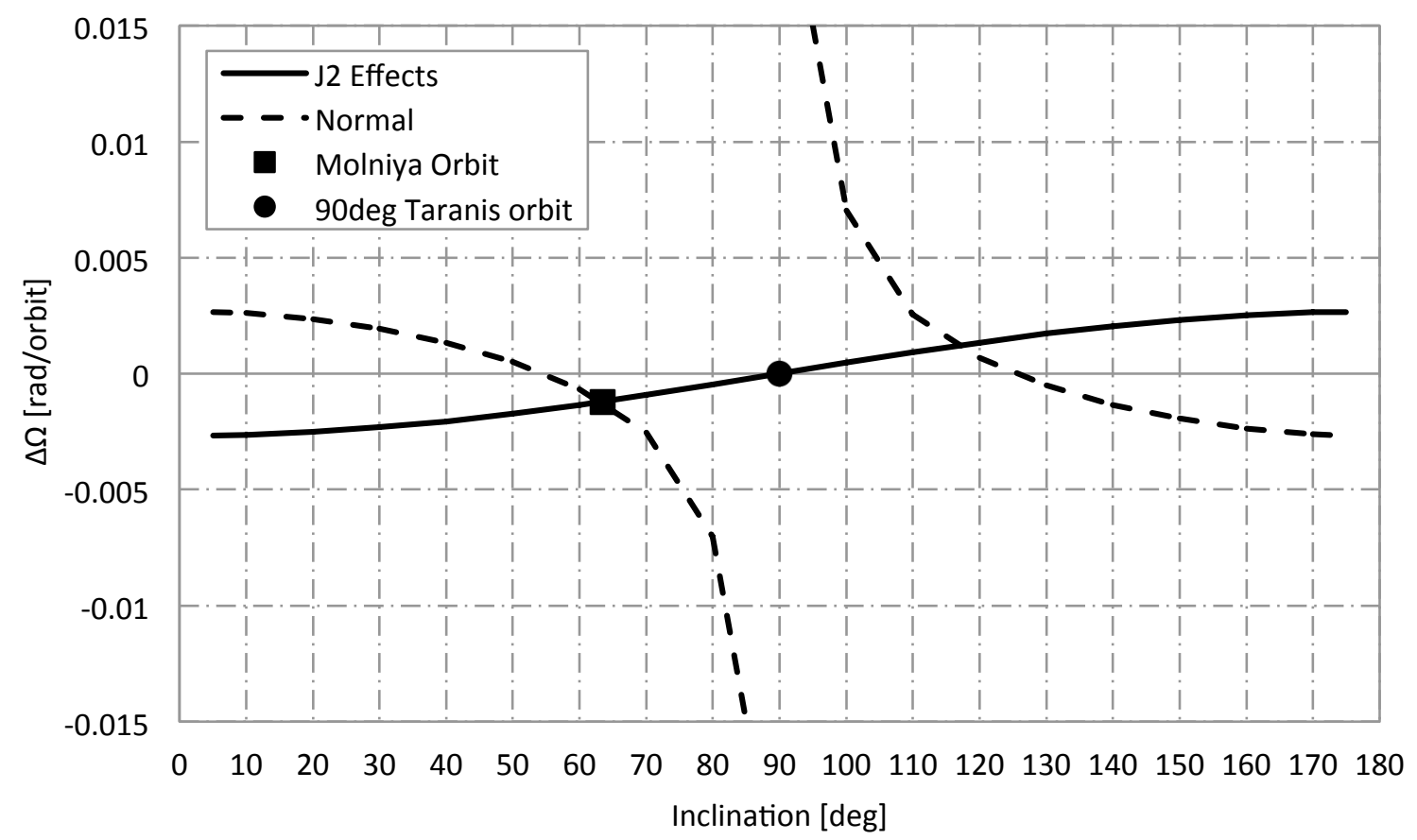

Fig. 3 Ascending node drift per orbit for extended HEOs and a Molniya orbit.

Most significantly, Fig. 3 shows that the 90deg, 12-hr Taranis orbit experiences no drift in ascending node under the influence of $J_{2}$ perturbations. It is also shown that between the inclination ranges of $5 \mathrm{deg}-70 \mathrm{deg}$ and $120 \mathrm{deg}$ $175 \mathrm{deg}$, Taranis orbits enabled using continuous normal acceleration have the same order of magnitude change in ascending node as a conventional Molniya orbit. However, as the inclination approaches 90deg, the change in ascending node increases rapidly and the singularity described in Fig. 1 for the normal acceleration cases is also observed in Fig. 3.

\section{Special Perturbations Technique}

To verify the general perturbations solution a special perturbations solution is generated. This numerical model propagates the spacecraft position by integrating a set of Modified Equinoctial Elements [17], using an explicit variable step size Runge Kutta $(4,5)$ formula, the Dormand-Price pair (a single step method) [18]. Numerical simulations include only perturbations due to Earth oblateness to the order of $J_{2}$. The numerical model proves that not only is the change in argument of perigee negligible due to the applied low-thrust, but the change in all other orbital elements also matches the analytical results. Fig. 4 shows the variation of semi-major axis, eccentricity and 
argument of perigee over seven orbital revolutions of the 12-hr Taranis orbit, inclined at 90deg to the equator, enabled by combined radial and transverse accelerations with a total magnitude of $0.0835 \mathrm{~mm} / \mathrm{s}^{2}$.

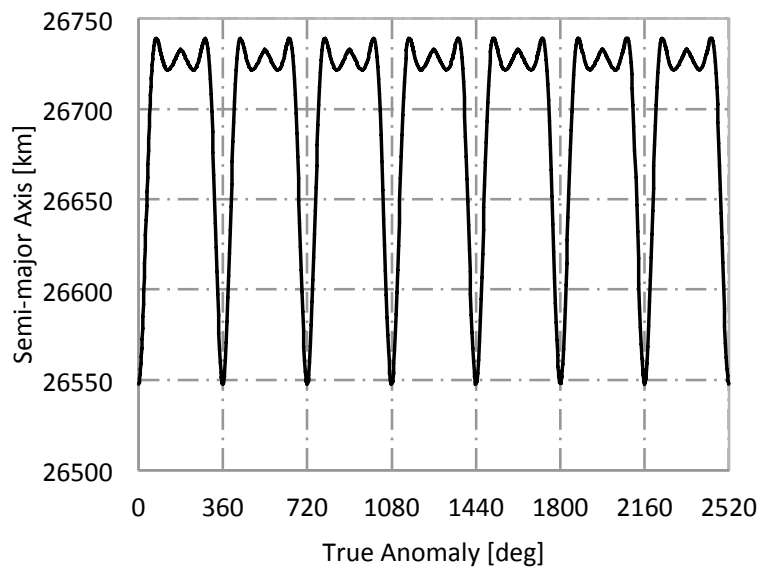

(a)

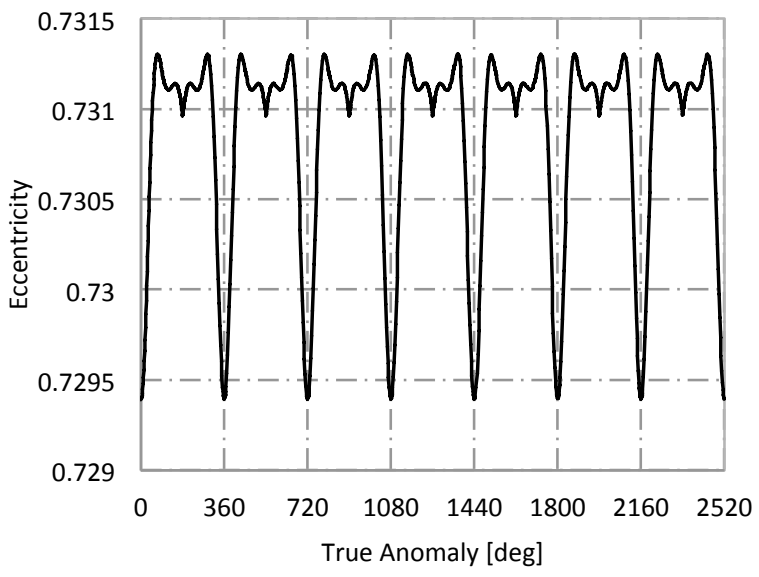

(b)

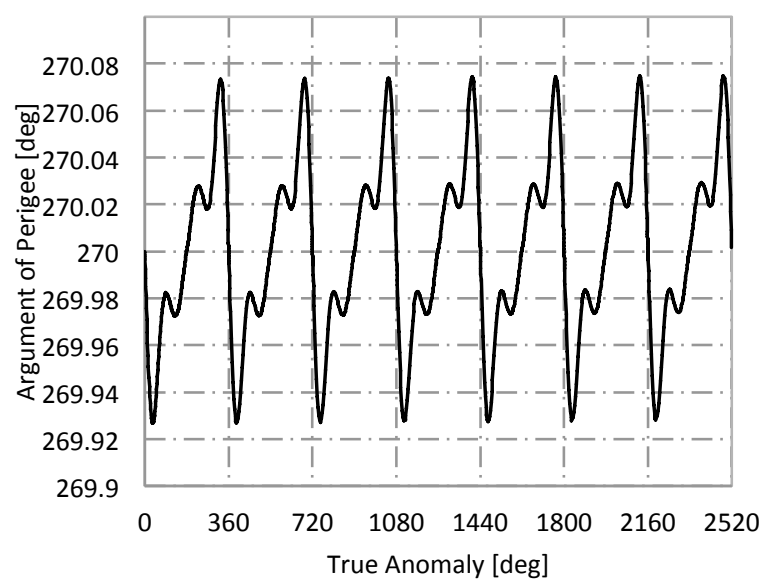

(c)

Fig. 4 Oscillation of orbital elements over seven orbital revolutions. (a) Semi-major axis. (b) Eccentricity.

(c) Argument of perigee.

Examination of Fig. 4 shows that although the semi-major axis, argument of perigee and eccentricity oscillate during one orbital period, all elements return to the same initial value. Examination of the inclination and ascending node angle revealed no oscillation over the orbital period as expected. 


\section{Optimization of the Taranis Orbit Control Profile}

As discussed previously, the problem becomes more complex when low-thrust normal to the orbit plane is included, meaning it is no longer possible to obtain a general perturbations solution. A Pseudospectral Optimal Control Solver (PSOPT) [19] is used to determine a numerically optimal solution combining low-thrust in all three axial directions in-order to quantify the optimality of the general perturbations solution. PSOPT uses a direct collocation method including pseudo-spectral and local discretization, to solve the optimal control problem by approximating the time-dependent variables using polynomials. The previous assumption that the fraction of the total acceleration is equal in each direction is no longer made, thus coupled with an objective function to maximize the final spacecraft mass generating a fuel optimal solution.

The results of the special perturbations solution are used as the initial guess for the optimizer, which once again propagates the spacecraft trajectory using a set of modified equinoctial elements [17]. The spacecraft initial mass is set to $1000 \mathrm{~kg}$, with a specific impulse of $4600 \mathrm{sec}$, and a total constant acceleration of $0.0835 \mathrm{~mm} / \mathrm{s}^{2}$. The analysis is initially conducted including perturbations due to the oblateness of the Earth to the order of $J_{2}$. This was subsequently extended to include $J_{3}$ and $J_{4}$, with no significant divergence found between the results. As such on order $J_{2}$ results are presented herein. The optimized control profiles in the radial, transverse and out-of-plane directions are shown in Fig. 5.

Each optimized solution was obtained using 17 nodes. It should be noted that due to the use of a variable step integrator within the special perturbations solution the initial guess does not simply contain equally spaced nodes and furthermore, due to the use of an adaptive mesh within the PSOPT tool the optimized nodes are not constrained by the initial guess. 


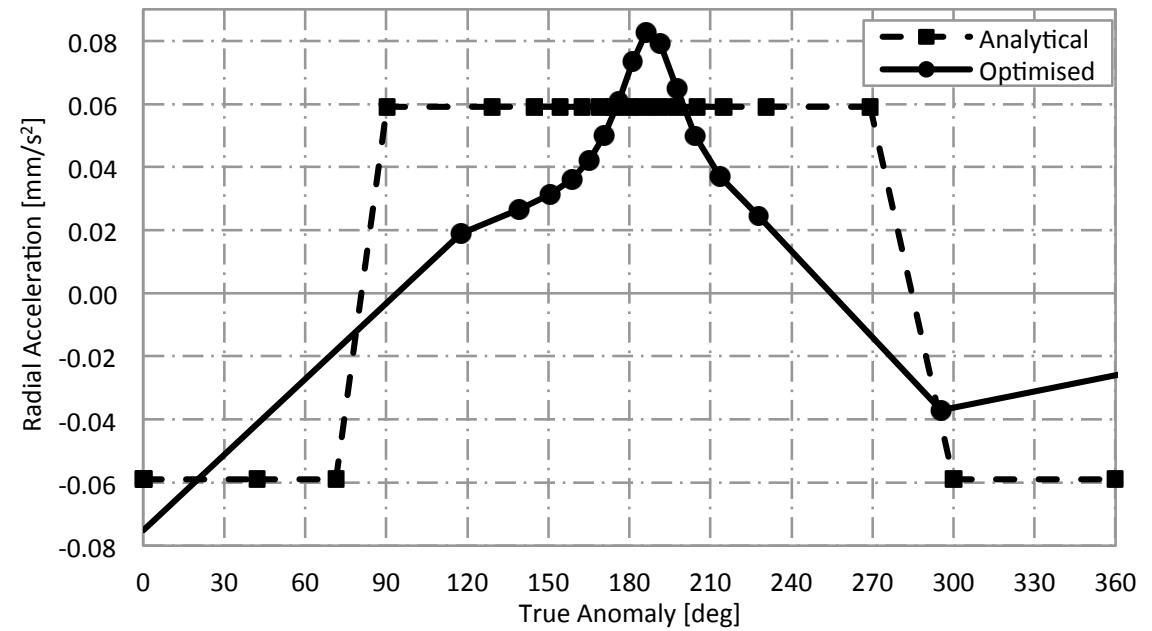

(a)

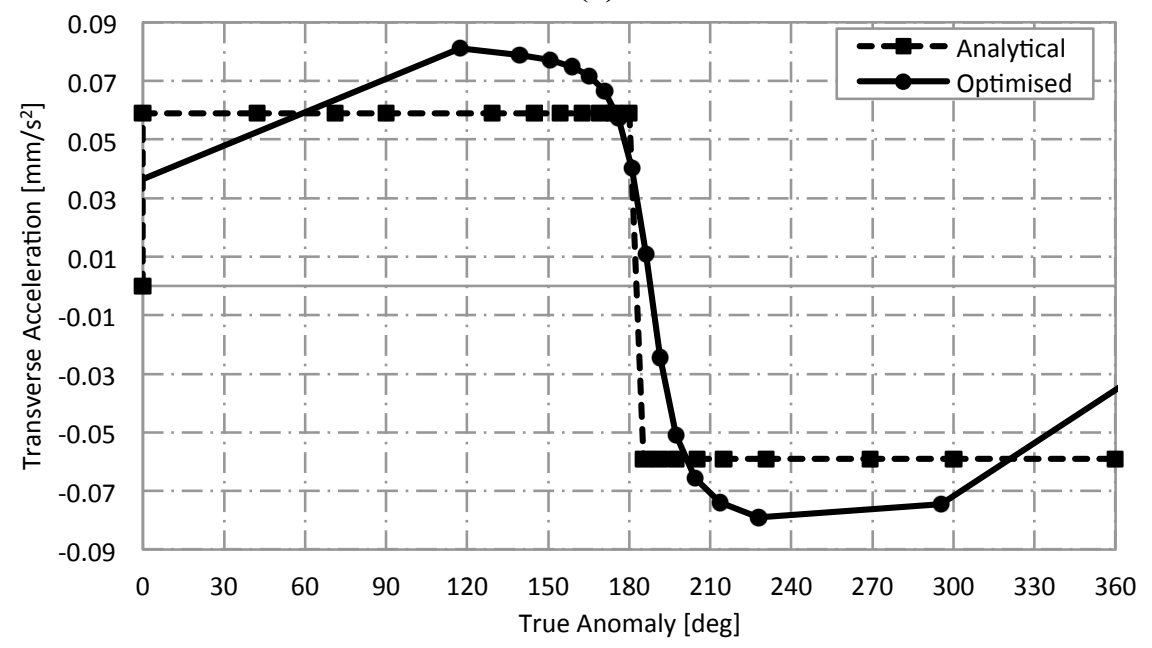

(b)

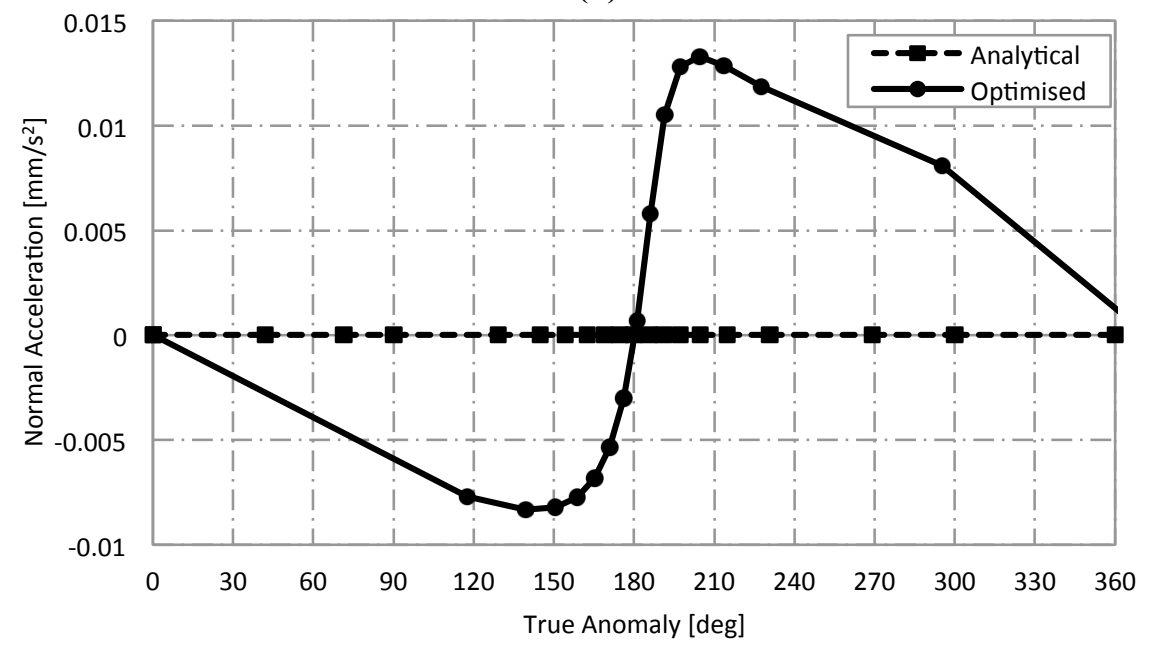

(c)

Fig. 5 Analytical and optimized control profiles for the 90deg. Taranis orbit, defined in Table 1. (a)

Radial control profile. (b) Transverse control profile. (c) Normal control profile. 
The control profiles show that as the assumption of equal acceleration magnitude is no longer made, the total acceleration is now composed of radial, transverse and out-of-plane components, with the fraction of the total acceleration in each direction varying with true anomaly. To complete one revolution of the Taranis orbit, the previous special perturbations technique required $80.5 \mathrm{~g}$ of fuel, while the fuel optimal solution consumes only $77.5 \mathrm{~g}$ of fuel, resulting in a reduction in fuel consumption of only $3.7 \%$. Given that the analytical solution is $<4 \%$ suboptimal this is likely to be considered an acceptable cost due to the significantly reduced complexity of flying a spacecraft using the general perturbations control profile over the optimized profile.

\section{Extended General Perturbations Solution}

Although the analysis throughout this paper considers the total acceleration magnitude from the general perturbations method assuming equal magnitudes of radial and transverse accelerations, the general perturbations solution can be extended to consider solutions where this assumption is no longer made. Thus, allowing the minimum acceleration magnitude to be determined.

Substituting the orbital element values from Table 1 into Eq. (15) and setting the change in argument of perigee equal to zero results in the following expression for the radial acceleration as a function of transverse acceleration,

$$
F_{r}=-1.58 e-4+1.676 F_{t}
$$

The transverse acceleration is assigned a range of values between $0 \mathrm{~mm} / \mathrm{s}^{2}$ and $0.1 \mathrm{~mm} / \mathrm{s}^{2}$, and the corresponding radial acceleration is found using Eq. (37). The results shown in Fig. 6 give a range of possible solutions for the 90deg Taranis orbit, while maintaining a constant argument of perigee. 


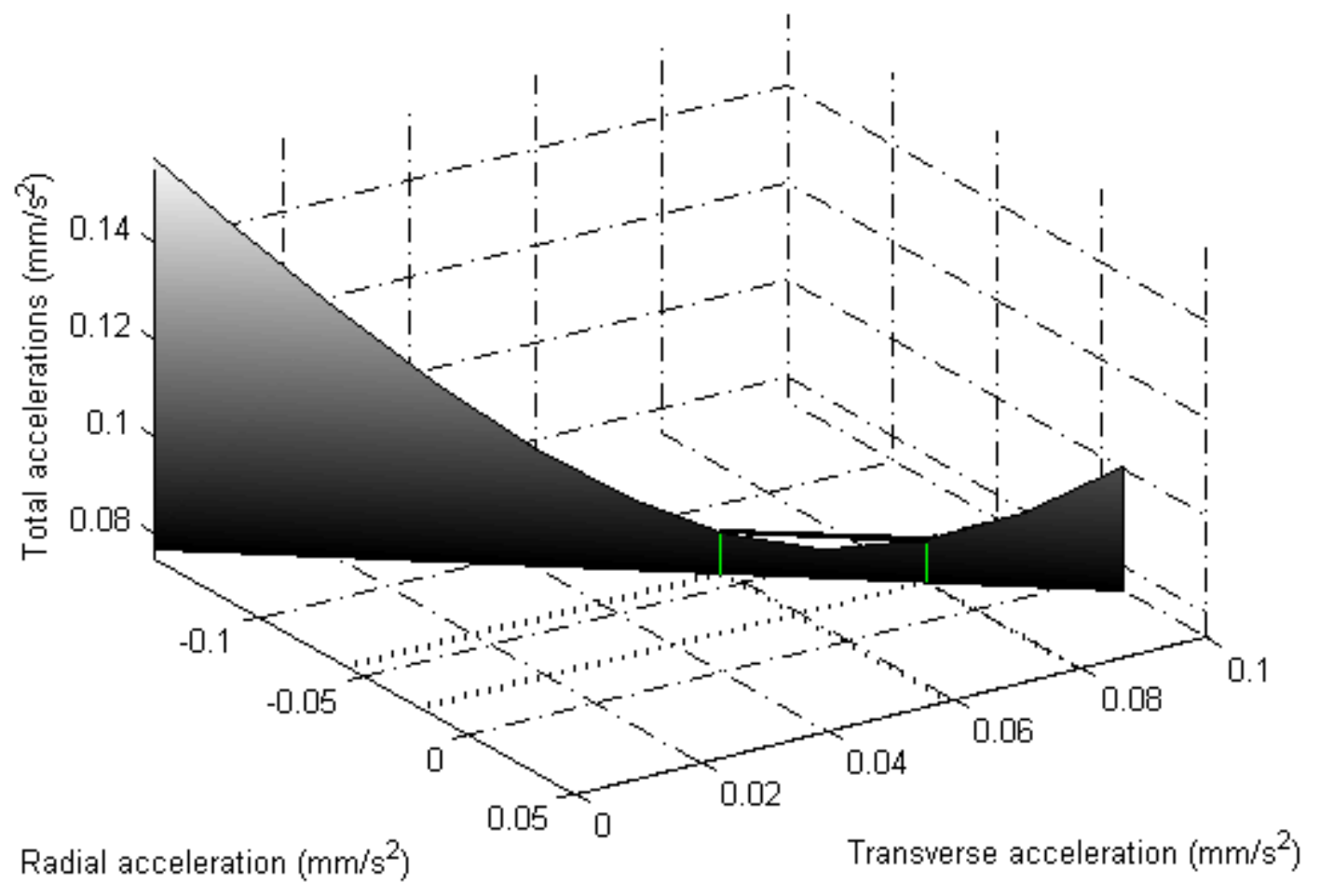

Fig. 6 Radial and transverse accelerations, total acceleration magnitude for zero change in argument of perigee.

Figure 6 shows the possible solutions for the Taranis orbit, for a range of transverse accelerations between $0 \mathrm{~mm} / \mathrm{s}^{2}$ and $0.1 \mathrm{~mm} / \mathrm{s}^{2}$, where between $F_{t}=0.059 \mathrm{~mm} / \mathrm{s}^{2}$ and $F_{t}=0.08 \mathrm{~mm} / \mathrm{s}^{2}$, the total acceleration magnitude of the solution is less than the previous general perturbations solution, i.e. using the assumption that the acceleration magnitudes are equal in both the radial and transverse directions. The previous general perturbations solution, where the two directional accelerations are equal in magnitude can also be seen in Fig. 6. It is also shown that the minimum acceleration magnitude occurs when the transverse acceleration is equal to $0.07 \mathrm{~mm} / \mathrm{s}^{2}$ and the corresponding radial acceleration is $-0.0406 \mathrm{~mm} / \mathrm{s}^{2}$ (from Eq. (37)). This results in a total acceleration magnitude of $0.0809 \mathrm{~mm} / \mathrm{s}^{2}$, around a $3 \%$ reduction from the previous general perturbations solution. This solution is validated using a special perturbations method and is again optimized to obtain the fuel optimal control profiles. This process, as with the previous optimization gives a $\sim 3.7 \%$ reduction in fuel consumption. This is again likely to be an acceptable cost due to the reduced complexity of flying the sub-optimal control profile. 


\section{Mission Analysis}

\section{Mission Lifetime}

By evaluating the performance of the Taranis orbit in terms of propellant consumption; possible mission lifetimes facilitated by means of Solar Electric Propulsion are determined. Defining the differential equation for the mass of the spacecraft as,

$$
\dot{m}=-\frac{T}{I_{s p} g_{0}}
$$

where $T$ is the thrust magnitude of the SEP system given by,

$$
T=a_{p} m
$$

Then making the appropriate substitution of Eq. (39) into Eq. (38) results in the following integral,

$$
\int_{m_{0}}^{m_{f}} \frac{d m}{m}=-\int_{t_{0}}^{t_{f}} \frac{a_{p}}{I_{s p} g_{0}} d t
$$

Evaluating the integrals and applying the condition that $\mathrm{t}_{0}=0$ gives the following expression for the mission lifetime,

$$
L=t_{f}=-\ln \left(\frac{m_{f}}{m_{0}}\right) \frac{I_{s p} g_{0}}{a_{p}}
$$

where the mass fraction $m_{f} / m_{0}$ is defined as,

$$
\frac{m_{f}}{m_{0}}=\frac{\left(m_{0}-m_{\text {prop }}\right)}{m_{0}}
$$

The lifetime of the 90deg 12-hr Taranis orbit detailed in Table 1 is thus determinable for a particular mass fraction and specific impulse. The resulting possible Taranis mission lifetimes are shown in Fig. 7. 


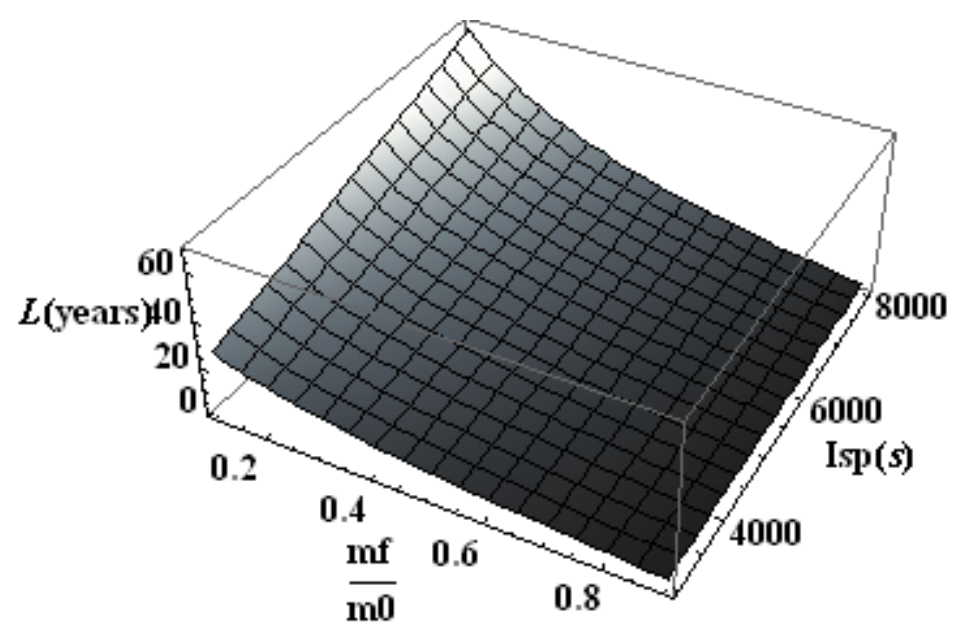

Fig. 7 Taranis mission lifetime.

Figure 7 shows that the possible mission lifetimes for the Taranis orbit range from around 1.2 years to around 69 years depending on the level of available technology and mass fraction; for example, using a specific impulse of 3000 s and fuel mass fraction of 0.5 results in a mission lifetime of approximately 8 years. Fig. 7 shows the results for a range of specific impulses from specific impulses possible with current technology to far term low-thrust propulsion technologies. For example, although the maximum mission lifetime shown is 69 years, for an $\mathrm{I}_{\mathrm{sp}}$ of 8000 s, current ion engines are not currently able to provide this level of specific impulse. Thus, to allow mission lifetimes of this order of magnitude, developments in low-thrust propulsion are required; such as JAXAs $\mu 10 \mathrm{HIsp}$ electric propulsion system, which has been under development since 2003, and will be capable of achieving a specific impulse of 10,000s [20]. As well as, NASAs Variable Specific Impulse Magnetoplasma Rocket (VASIMR) currently under development will be capable of providing between 5,000s and 50,000s of specific impulse

\section{Mass Budget}

Although the mission lifetime analysis characterizes possible mission lifetimes of the Taranis mission in terms of propellant consumption, it should also be investigated whether these conditions allow a useful payload to be carried. The initial mass of the spacecraft, $m_{0}$, is composed of many elements and subdivided as,

$$
m_{0}=m_{\mathrm{sys}}+m_{\mathrm{prop}}+m_{\mathrm{tank}}+m_{\mathrm{SEP}}+m_{\mathrm{P}}+m_{\mathrm{pay}}
$$

\footnotetext{
\$ http://web.mit.edu/mars/Conference_Archives/MarsWeek04_April/Speaker_Documents/VASIMREngineTimGlover.pdf
} 
where the system mass includes, for example, telecommunications, data processing, guidance, navigation and control, structural mass and any power system requirements beyond the SEP systems requirements. Throughout the analysis, this system mass is assumed to total $500 \mathrm{~kg}$ and the specific impulse is assumed to be 3000s. From Eq. (43), $m_{\text {prop}}$, is the mass of the available propellant for a given mission duration, found using the difference between the initial spacecraft mass and the final spacecraft mass, $m_{f}$. The final mass is approximated as,

$$
m_{\mathrm{f}}=m_{0}-\frac{T_{\max }}{I_{s p} g_{0}} \Delta t
$$

Thus, the mass of propellant over a given time, $\Delta t$ is

$$
m_{\text {prop }}=\frac{T_{\max }}{I_{S P} g_{0}} \Delta t
$$

The mass of the propellant tanks, $m_{\text {tank }}$, is found as a function of the mass of the propellant, as $m_{\text {tank }}=0.1 m_{\text {prop }}$. The mass of the SEP thruster, $m_{S E P}$ is then found as a function of the maximum power provided by the SEP system, $P_{\max }$, and is given by,

$$
m_{\mathrm{SEP}}=k_{S E P} P_{\max }
$$

With the specific performance of the thruster given as $k_{S E P}=0.02 \mathrm{~kg} / W$ [21]. The thruster efficiency, $\eta_{S E P}$ is assumed to be equal to 0.7 . In Eq. (43) $m_{p}$ is the mass of the spacecraft power system required to provide electrical energy to the SEP system. Thus, using a solar array the mass is given by,

$$
m_{\mathrm{P}}=k_{S A} P_{\max }
$$

Using a conservative estimate of the specific performance of the solar array, from [22], of $k_{s a}={ }^{1} /{ }_{45} \mathrm{~kg} / \mathrm{W}$. The initial mass of the spacecraft is found as a function of maximum thrust, Eq. (39) the maximum allowable initial mass is thus determined for the given level of thrust, using a constant SEP acceleration of $0.0835 \mathrm{~mm} / \mathrm{s}^{2}$, as illustrated in Fig. 8 . 


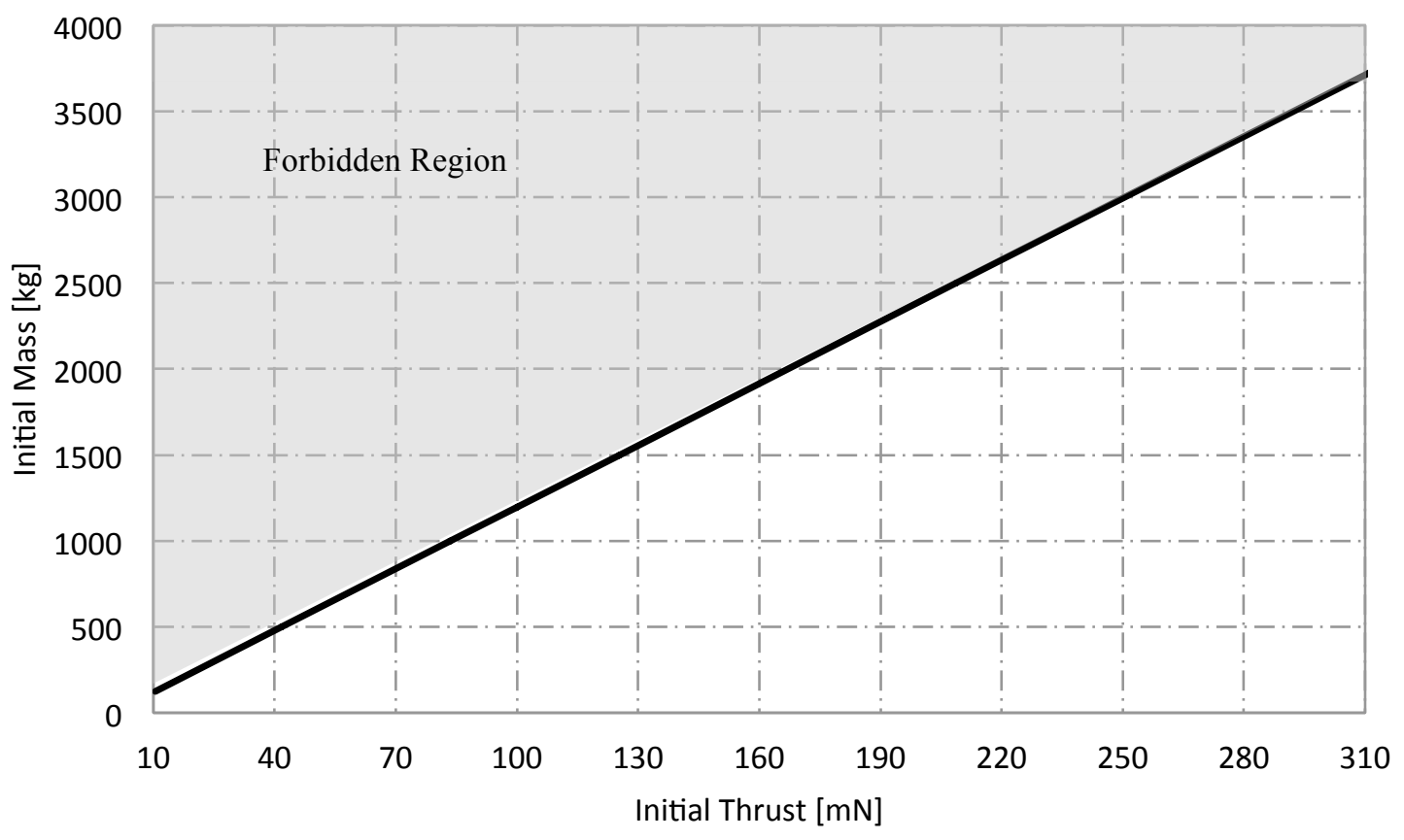

Fig. 8 Maximum allowable initial mass for the 12-hr Taranis orbit in Table 1.

Figure 8 shows the maximum possible initial mass of the spacecraft for a range of thrust values between $10 \mathrm{mN}$ and $310 \mathrm{mN}$, where the shaded region above the line gives the spacecraft masses that cannot be achieved for a given level of thrust for the constant acceleration considered. It can be seen, from Fig. 8, that for spacecraft with maximum thrust values of $83.5 \mathrm{mN}, 125.3 \mathrm{mN}$, and $208.8 \mathrm{mN}$, the maximum allowable initial mass is $1000 \mathrm{~kg}$, $1500 \mathrm{~kg}$, and $2500 \mathrm{~kg}$ respectively. Inserting the appropriate values into Eq. (43), and solving for the payload mass, $m_{p a y}$, for a range of mission lifetimes results in Fig. 9. 


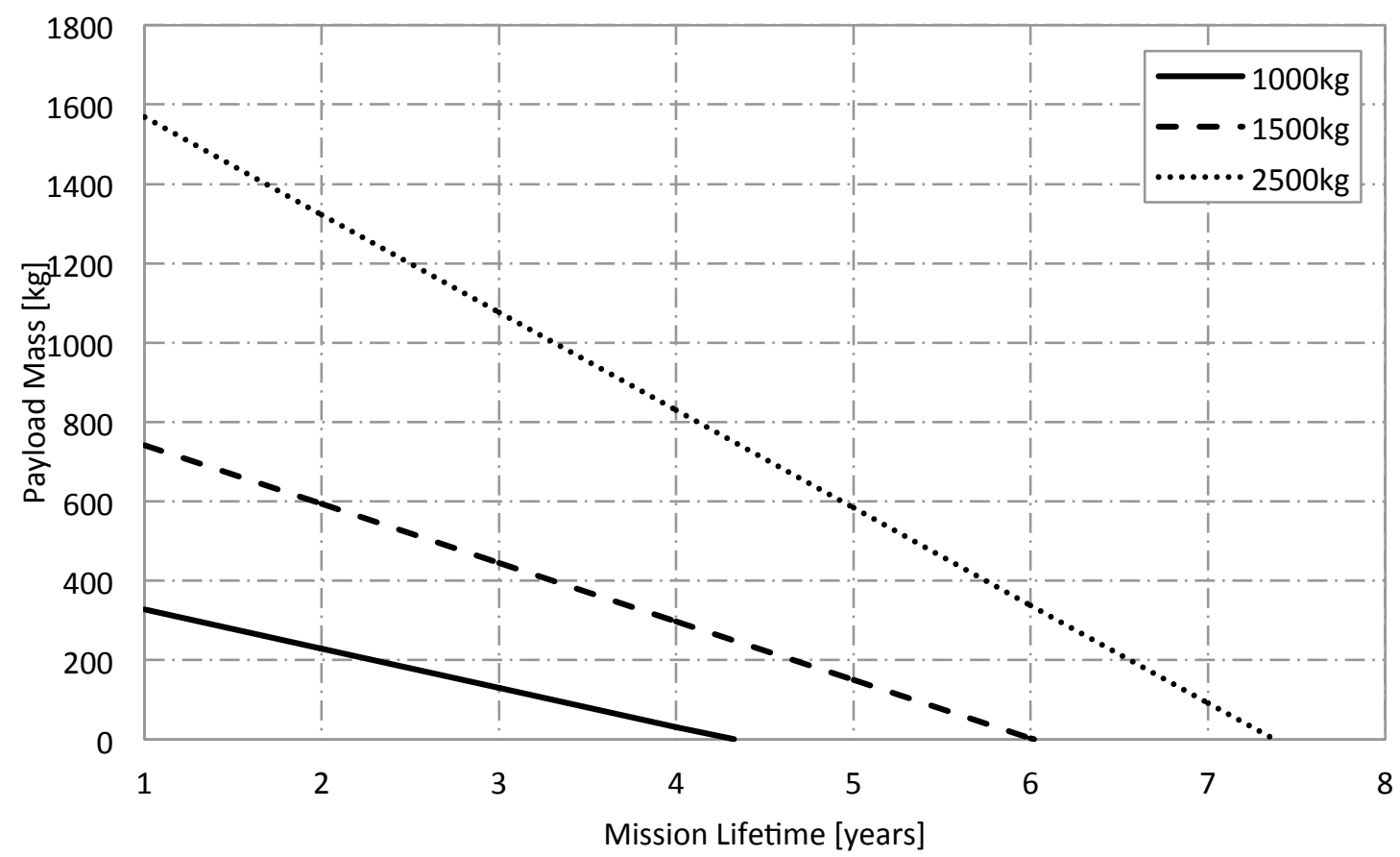

Fig. 9 Payload mass as a function of mission lifetime.

Figure 9 shows the payload mass for each initial mass of spacecraft as a function of mission lifetime and allows the maximum mission lifetime to be determined, that is, where there is no longer any capacity for useful payload. This results in maximum mission lifetimes of 4.3 years, 6 years and 7.4 years respectively for each initial mass of spacecraft considered. Although mission lifetimes were outlined in Fig. 7 in terms of propellant consumption, Fig. 9 shows that the actual mission lifetimes are significantly reduced when the availability for useful payload is considered. The mission lifetimes given in Fig. 9 for the $1500 \mathrm{~kg}$ and $2500 \mathrm{~kg}$ spacecraft are around the values expected for Molniya-like orbits [23]. However, the lifetime shown for the 1000kg spacecraft is lower, nonetheless a $1000 \mathrm{~kg}$ spacecraft is at the lower end of Earth Observation spacecraft currently being launched, and so the lower lifetime for this mass of spacecraft is of little concern.

\section{Thrust Range Analysis}

Altering the inclination to values other than the conventional critical inclinations requires an acceleration of constant magnitude to compensate for the drift in argument of perigee caused by Earth's gravitational field. However, in reality as the propellant is consumed, the mass of the spacecraft decreases consequently a variable thrust SEP system is required. The thrust range necessary from the SEP system can be determined by finding the thrust at the beginning of the mission with all the propellant, and the thrust at the end of the mission with zero 
propellant. These thrust ranges are shown for the three initial mass of spacecraft previously considered, for a range of mission lifetimes, shown in Fig. 10.

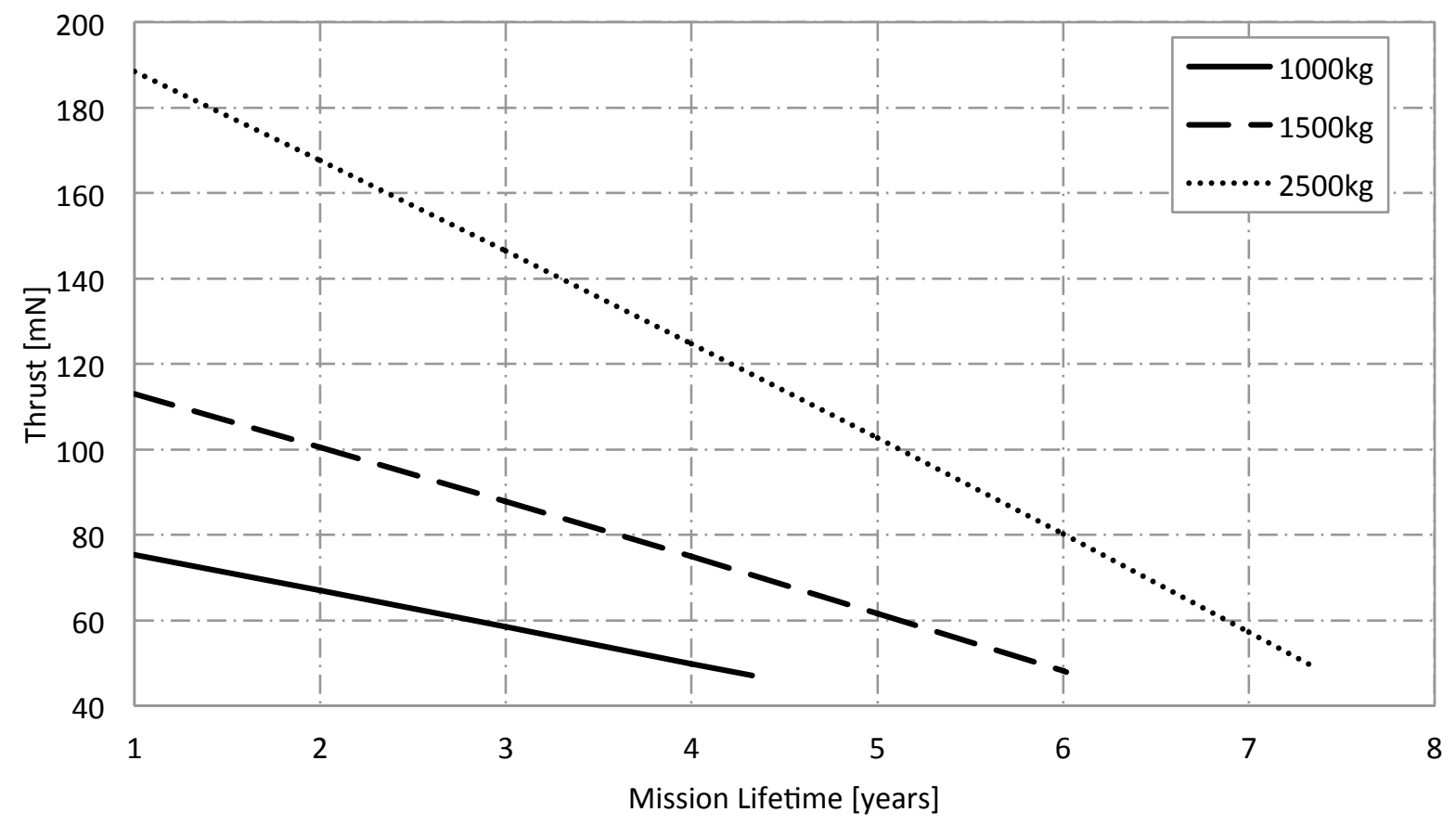

Fig. 10 Thrust ranges required for SEP system.

Considering the specific case of the $1000 \mathrm{~kg}$ spacecraft with mission duration of 4 years, the thrust at the beginning of the mission with all the propellant is $83.5 \mathrm{mN}$ and at the end of the mission with all propellant consumed is $49.9 \mathrm{mN}$.

\section{Technology Requirements}

Given that the parameters of the Taranis platform are defined for the mass and power required, the systems and technology requirements for the platform can be investigated. Firstly, the requirements of SEP thruster are discussed. Assuming the total acceleration is constituted by two thrusters at any given time, one for each of the radial and transverse directions, the approximate required range per thruster for the three specified initial masses of spacecraft for given mission durations, are stated in Table 2. 
Table 2 Maximum and minimum thrust per thruster.

\begin{tabular}{cccc}
\hline \hline & & & \\
Initial Mass [kg] & Mission Duration [years] & Maximum Thrust [mN] & Minimum Thrust [mN] \\
\hline 1000 & 4 & 42 & 25 \\
1500 & 6 & 63 & 24 \\
2500 & 7 & 104 & 29 \\
\hline \hline
\end{tabular}

NASA's Solar Electric Propulsion Technology Application Readiness (NSTAR) thruster, which has undergone significant ground testing in addition to a flight test on the Deep Space 1 (DS1) spacecraft [24], is capable of providing between $20 \mathrm{mN}$ and $94 \mathrm{mN}$ of thrust. Thus, four NSTAR thrusters, one per required direction, are capable of providing the thrust range required for the both the $1000 \mathrm{~kg}$ spacecraft and the $1500 \mathrm{~kg}$ spacecraft. Furthermore, the QinetiQ T6 thruster, is throttelable between $30 \mathrm{mN}$ and $210 \mathrm{mN}$ and is thus capable of providing the required thrust range of the $2500 \mathrm{~kg}$ spacecraft [25].

To enable the 90deg Taranis orbit, the power requirements of the spacecraft must be considered. The sizing of the required solar arrays is based on an end-of-life (EOL) solar array efficiency of 0.25 at 1 AU. The power required by each spacecraft, the mass of the solar arrays (from Eq.(46)) and the required solar array area, found using the Solar flux of $1370 \mathrm{~W} / \mathrm{m}^{2}$ at $1 \mathrm{AU}$, are given in Table 3 . Where, it is shown that the required sizes of the solar arrays are modest and feasible using current solar array technology. With the solar arrays of Rosetta totaling $61.5 \mathrm{~m}^{2}$ [26] and SMART-1's arrays having an area of $10 \mathrm{~m}^{2}$ [27] for spacecraft of $3000 \mathrm{~kg}$ and $370 \mathrm{~kg}$ respectively.

Table 3 Solar array sizing.

\begin{tabular}{cccc}
\hline \hline Initial Mass [kg] & Maximum Power [kW] & Solar Array Mass [kg] & Solar Array Area [m ${ }^{2}$ ] \\
\hline 1000 & 1.8 & 39 & 5 \\
1500 & 2.6 & 58 & 8 \\
2500 & 4.4 & 97 & 13 \\
\hline \hline
\end{tabular}

Finally, the storage requirements for the requisite propellant mass for a given duration are examined. For the maximum mission durations for each initial mass of spacecraft discussed in the previous section the mass of propellant is determined using Eq. (45) and shown in Table 4.

Table 4 Propellant mass.

\begin{tabular}{ccc}
\hline & & \\
Initial Mass [kg] & Mission Duration [years] & Propellant Mass [kg] \\
\hline 1000 & 4 & 358 \\
1500 & 6 & 806 \\
2500 & 7 & 1566 \\
\hline \hline
\end{tabular}


The NASA Dawn mission Xenon tanks have a capacity of $425 \mathrm{~kg}$ of propellant, thus the Taranis spacecraft propellant mass requirements can be accommodated using one, two and four tanks of comparable capacity, respectively for each of the three spacecraft. The single tank volume is $0.27 \mathrm{~m}^{3}$, so that the equivalent tank radius for a spherical tank is $0.4 \mathrm{~m}$, thus the total propellant mass requirements for the possible Taranis platforms can be accommodated in a modest volume.

\section{Radiation Analysis}

A brief radiation analysis is also conducted from The Space Environment Information System (SPENVIS) $^{\S}$, the results of which are shown in Fig. 11.

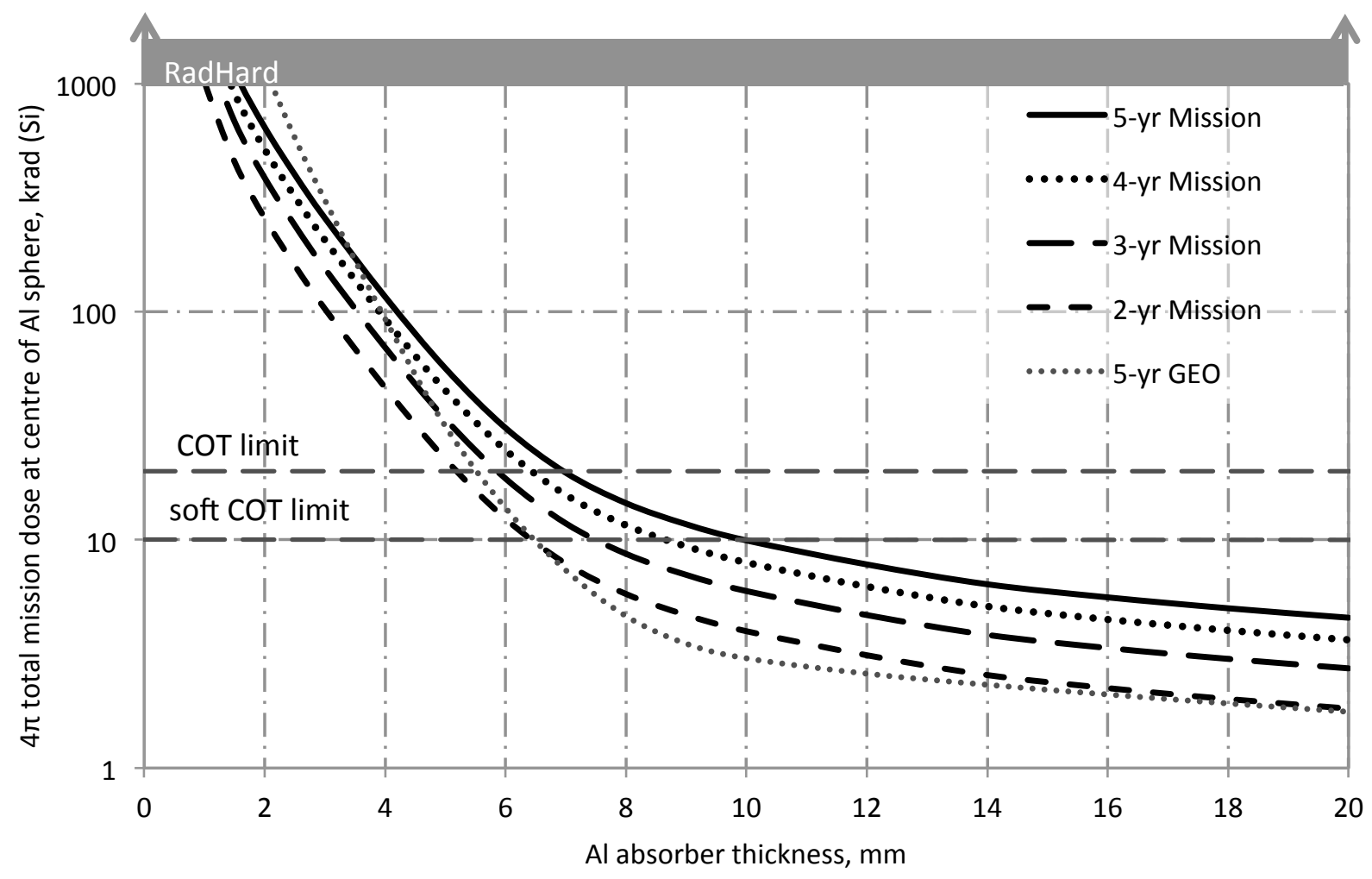

Fig. 11 Radiation analysis.

Figure 11 shows the total radiation experienced by a Taranis spacecraft for mission durations between 2 and 5 years for various Aluminum absorber thicknesses. Using a typical absorber thickness of $4 \mathrm{~mm}$ for all mission lifetimes it is necessary that the Taranis platform hardware must be space qualified. However, the values of radiation

\footnotetext{
$\S$ www.spenvis.oma.be/
} 
are below those requiring radiation hard materials and are of the same order of magnitude as the radiation levels experienced by existing geostationary spacecraft, again demonstrating the viability of the Taranis mission using current technology

\section{Visibility Analysis}

The number of spacecraft required to provide continuous observation of the Frigid and neighboring Temperate zones, to 55deg latitude, using both the Molniya and 12-hr Taranis orbit are determined. The continuous visibility analysis is achieved by determining the time that spacecraft on both the Molniya orbit and the Taranis orbit can view ground stations located at longitude intervals of $10 \mathrm{deg}$ at the desired latitude limit. This is accomplished by assuming a value of the minimum elevation and calculating the maximum elevation angle (parameters are introduced in Fig. 12) for each ground station in turn; between these elevation ranges the spacecraft is in view of the ground station. The coverage for a number of spacecraft over time is then plotted to determine the minimum number of spacecraft required to achieve continuous coverage of the given region. In this instance the region under consideration is above $55 \mathrm{deg}$ latitude, due to the limitations of geostationary platforms beyond this.

A relatively high minimum elevation angle is assumed throughout the visibility analysis; the basis for this assumption is the requirement to match the OZA of the defined latitude limit when viewed from geostationary orbit. Considering a geostationary spacecraft at an altitude of $36,000 \mathrm{~km}$ viewing $55 \mathrm{deg}$ latitude, the minimum elevation angle is determined using the following relationships. Firstly, the angular radius of the Earth is calculated using,

$$
\sin \rho=\cos \lambda_{0}=\frac{R_{E}}{R_{E}+H}
$$

and the nadir angle is determined as,

$$
\tan \eta=\frac{\sin \rho \sin \lambda}{1-\sin \rho \cos \lambda}
$$

The angular radius of the Earth and nadir angle are used to obtain the minimum elevation angle using,

$$
\cos \varepsilon=\frac{\sin \eta}{\sin \rho}
$$


The geostationary spacecraft thus has a minimum elevation angle of $27 \mathrm{deg}$, or an equivalent OZA of $63 \mathrm{deg}$. Employing this minimum elevation angle for both the Molniya and Taranis orbits ensures that these provide at the very least data of equal quality to that produced by geostationary systems.

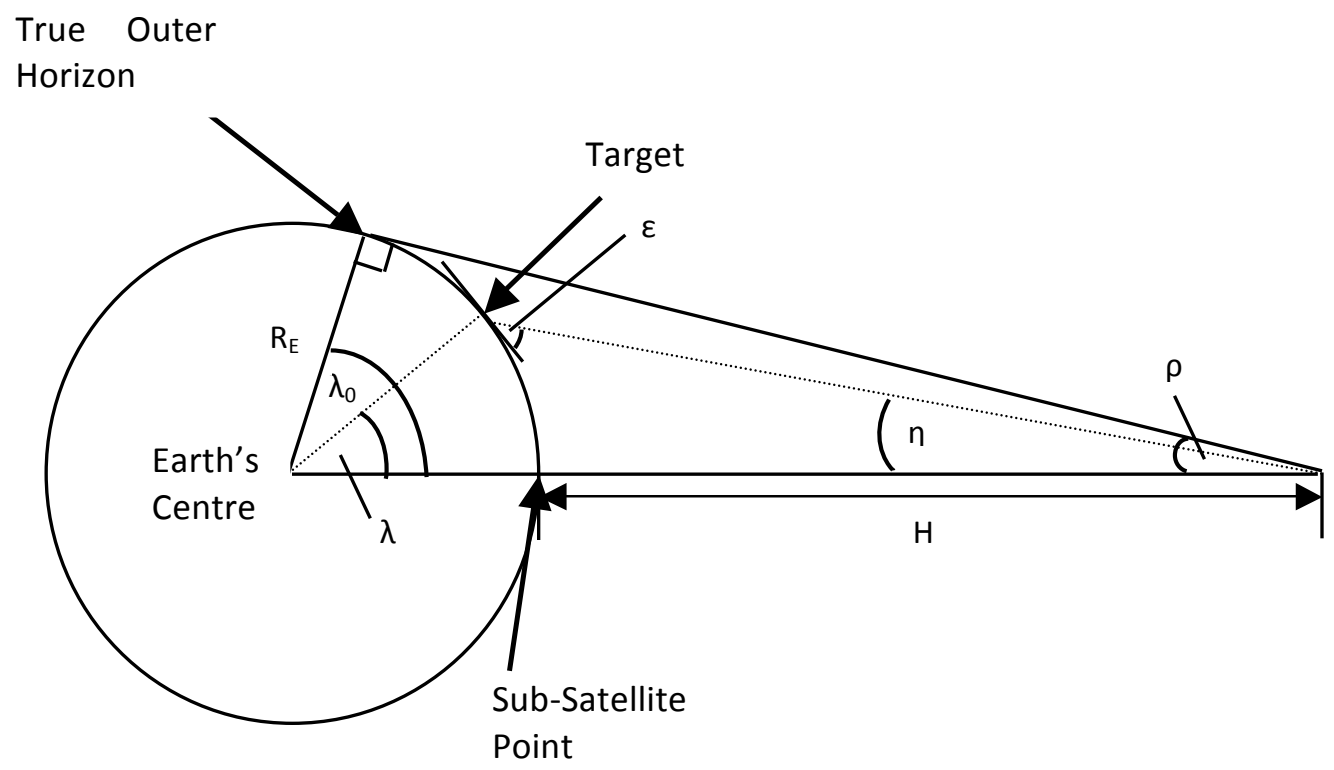

Fig. 12 Angular relationships between satellite, target and Earth center.

Conducting the analysis for the given visibility conditions, demonstrates that the Molniya orbit is, from a single platform, unable to provide any coverage to $55 \mathrm{deg}$ latitude at the desired observational zenith angle. Observation to $55 \mathrm{deg}$ latitude from the Molniya orbit only begins to become feasible at an observational zenith angle of around 69deg. However, this observational zenith angle is clearly too high for imaging of equal quality to that of geostationary systems. This highlights the requirement for an orbit capable of providing accurate imaging of high latitude regions. The 90deg inclination Taranis orbit is capable of providing continuous observation of this region using a constellation of only three spacecraft, as shown in Fig. 13. A possible configuration of such a Taranis constellation is demonstrated in Fig. 14. 


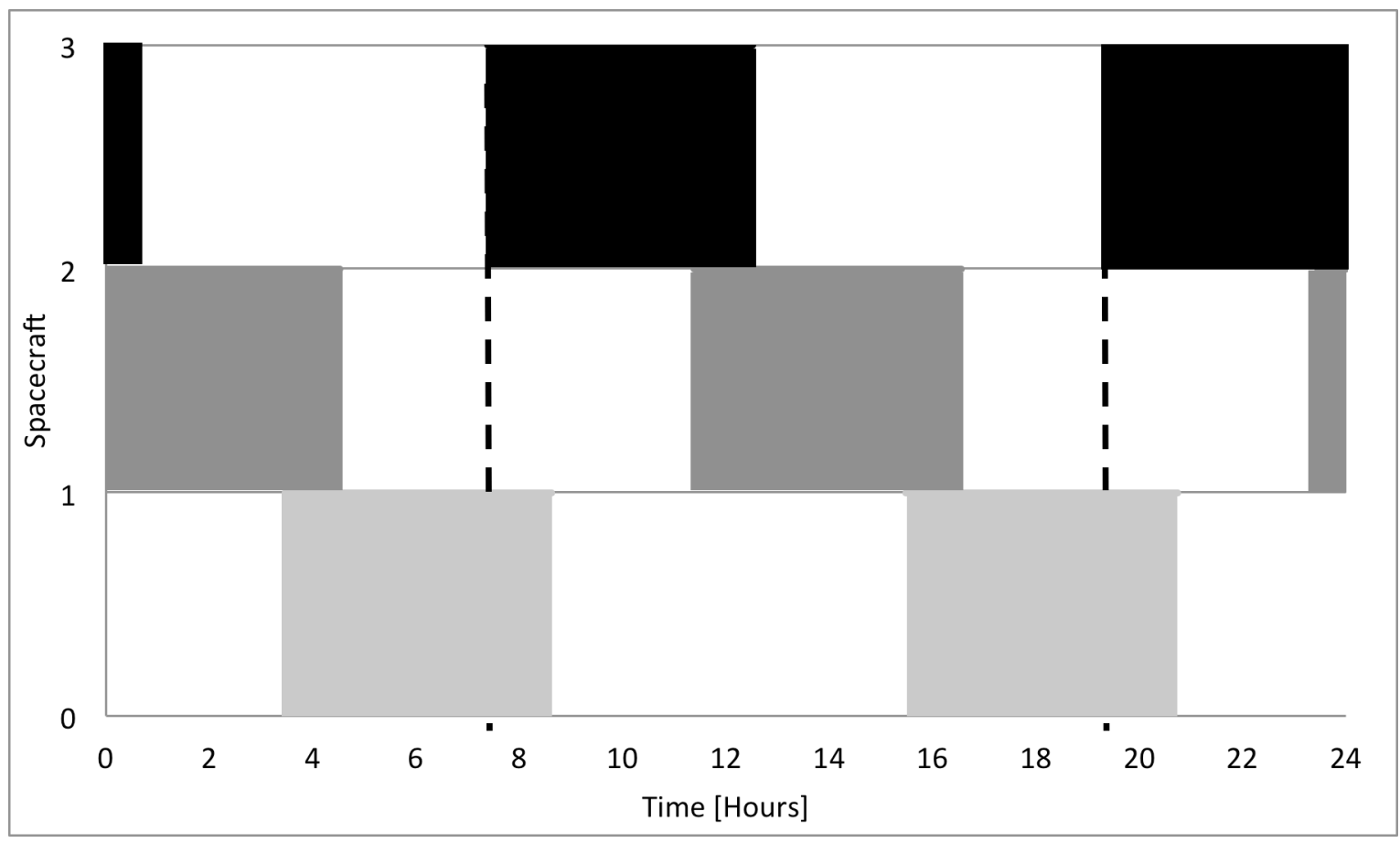

Fig. 13 Taranis orbit observation above 55deg latitude. 


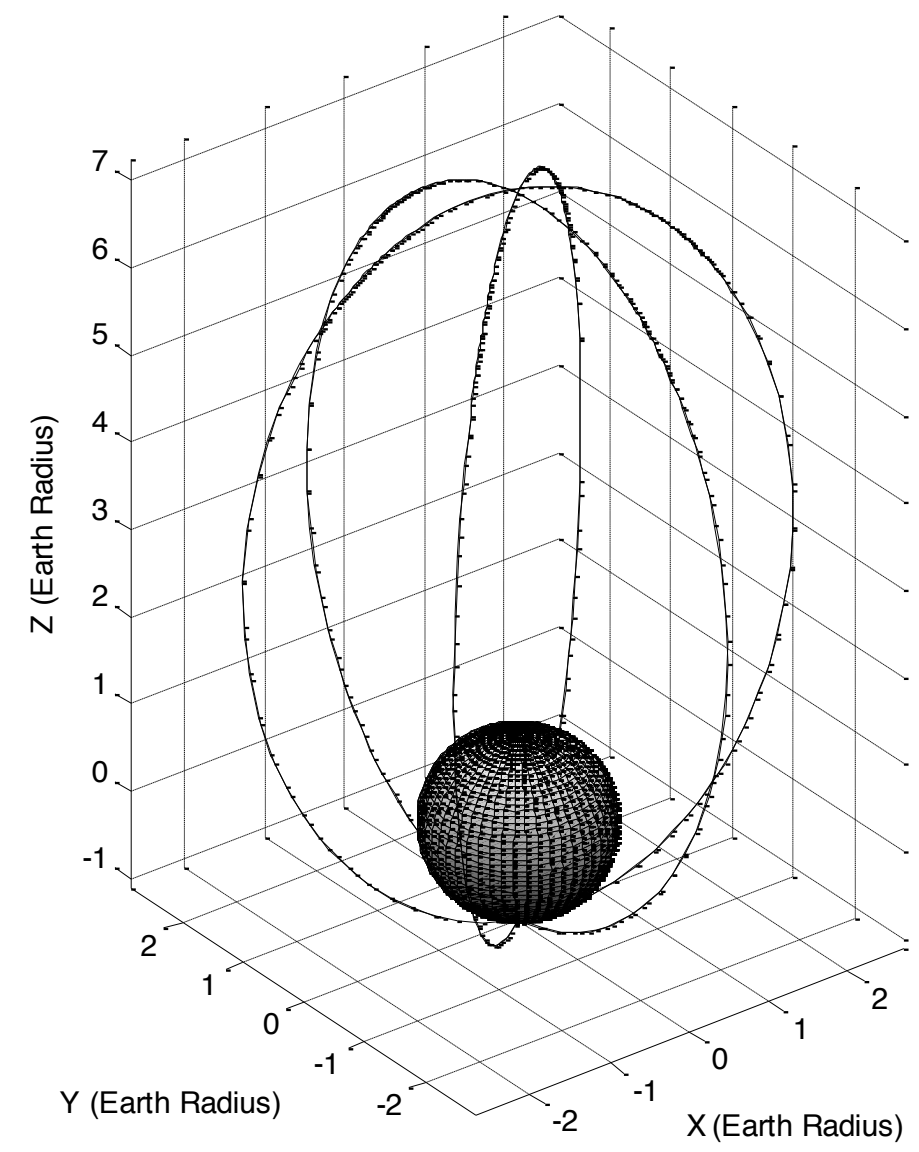

Fig. 14 Taranis orbit, three spacecraft constellation.

It is important to note that while Fig. 14 shows the constellation of spacecraft on the Taranis orbit at equally spaced ascending node values, this parameter has no effect on the coverage delivered. As such each spacecraft could have the same ascending node allowing them to be launched on a single launch vehicle, thus reducing the overall cost.

\section{Electric and Chemical Propulsion Comparison}

The effective $\Delta v$ to enable the Taranis orbits using continuous low-thrust propulsion can be found from the product of the required acceleration and mission duration, given by,

$$
\Delta v=a_{p} \Delta t
$$

Comparison can thus be made with the use of high-thrust chemical propulsion to maintain the artificial critical inclination of the Taranis orbits. At an inclination of $90 \mathrm{deg}$ without any control the drift in argument of perigee is 
minus 0.15 deg per day. To compensate for this change in argument of perigee chemical thrusters would be required to provide two equal impulses per orbit. The total $\Delta v$ through the application of the two impulses is given by Eq.(52) [28],

$$
\Delta v=\frac{\Delta \omega}{(2 / e) \sqrt{a\left(1-e^{2}\right) / \mu}}
$$

The respective $\Delta v$ and propellant mass fraction, $\Delta m / m_{0}$, calculated using the rocket equation, for one year of operation are shown in Table 5, with the results extended over various mission durations shown in Fig. 15.

Table 5. $\Delta v$ requirements for one year of operation.

\begin{tabular}{ccc}
\hline \hline Propulsion System & $\boldsymbol{\Delta} \boldsymbol{v}[\mathbf{k m} / \mathbf{s}]$ & Propellant mass fraction \\
\hline Electric $\left(\mathrm{I}_{\mathrm{sp}}=3000-4600 \mathrm{~s}\right)$ & 2.63 & $0.086-0.057$ \\
Chemical $\left(\mathrm{I}_{\mathrm{sp}}=200-340 \mathrm{~s}\right)$ & 1.96 & $0.632-0.444$ \\
\hline \hline
\end{tabular}

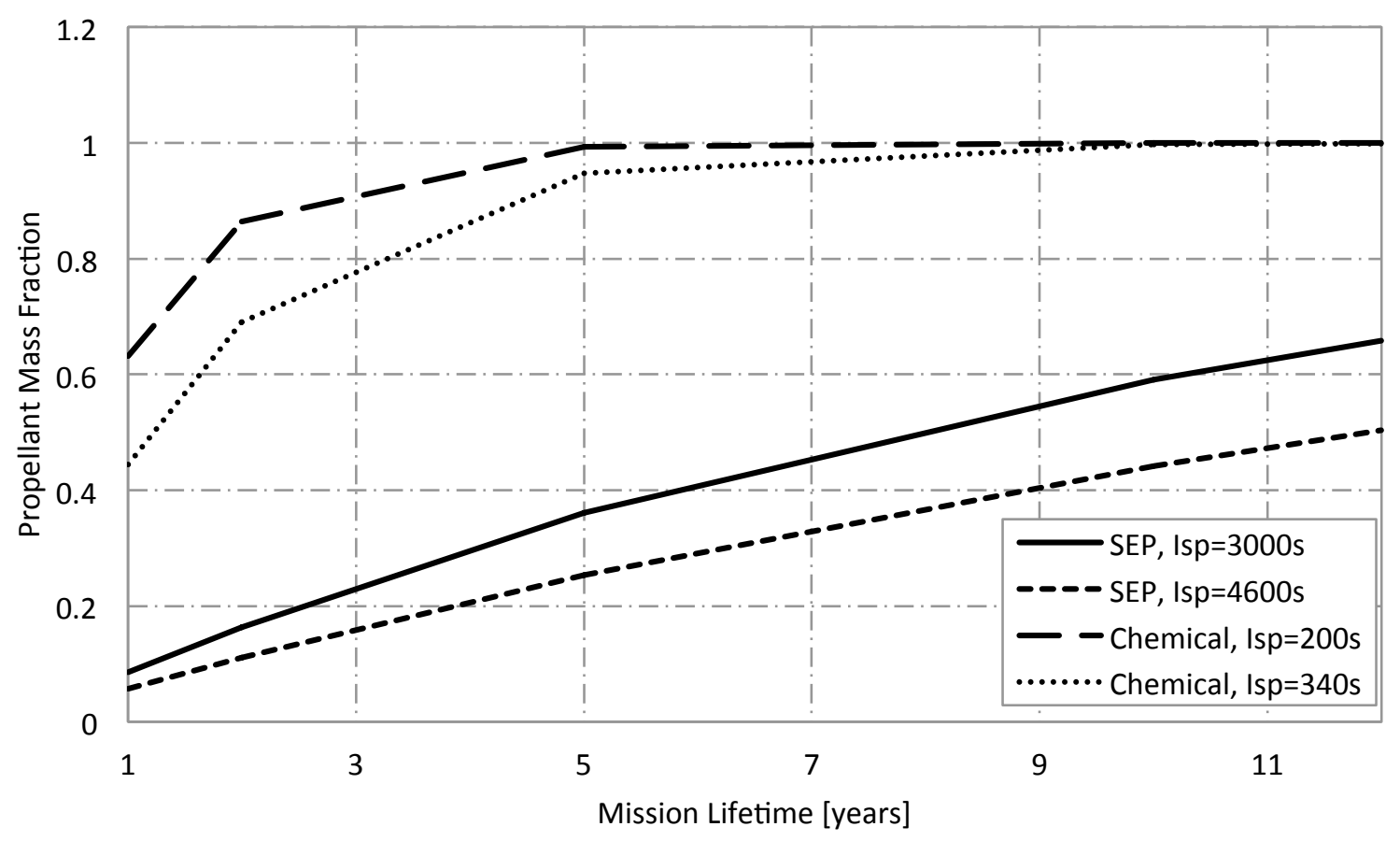

Fig. 15 Electric and chemical $\Delta v$ comparison.

Figure 15 highlights the benefit of utilizing SEP to enable Taranis orbits, as chemical propulsion, although feasible for low mission durations becomes impractical for longer duration missions. Above 5 year missions the 
chemical propellant mass fraction begins to tend to one, with low-thrust propulsion enabling much greater mission lifetimes.

\section{Conclusion}

The use of continuous low-thrust propulsion to extend conventional highly elliptical Earth orbits has been demonstrated. It is shown that low-thrust propulsion can be used to alter the natural critical inclination of Molniyalike orbits to any inclination to optimally fulfill the mission goals, independent of the orbit period, perigee or apogee altitudes. These low-thrust extensions are termed Taranis orbits. The most significant finding is a Taranis orbit inclined at 90deg to the equator, with a constellation of three spacecraft on this orbit enabling high latitude imaging previously unfeasible using existing Earth orbits. Notably, these new Taranis orbits can be enabled with existing technology.

\section{References}

1. Boain, R. J. "A-B-Cs of Sun-Synchronous Orbit Mission Design,"14th AAS/AIAA Space Flight Mechanics Conference,Maui, Hawaii,2004,8-12 February 2004

2. Lazzara, M. A., Keller, L. M., Strearns, C. R., Thom, J. E., and Wieder, G. A. "Antartic Satellite Meteorology; Applications for Weather Forecasting," Monthly Weather Review Vol. 131, 2003, pp. 371

3. Wertz, J. R., "Mission Geometry: Orbit and Constellation Design and Management", The Space Technology Library, ed., Vol., 2001. p. 619.

4. $\quad$ McInnes, C. R. "The Existence and Stability of Families of Displaced Two-Body Orbits," Celestial Mechanics and Dynamical Astronomy Vol. 67, 1997, pp. 167May 1997

5. McInnes, C. R. "Dynamics, Stability, and Control of Displaced Non-Keplerian Orbits," Guidance, Control and Dynamics Vol. 21, No. 5, 1998, pp. 799September-October 1998

6. McKay, R., Macdonald, M., Biggs, J., and McInnes, C. R. "Survey of Highly Non-Keplerian Orbits With Low-Thrust Propulsion," Journal of Guidance, Control and Dynamics Vol. 34, No. 3, 2011, pp. 645

7. Heiligers, J. "Displaced Geostationary Orbits using Hybrid Low-Thrust Propulsion,"61st International Astronautical Congress,Prague, CZ,2010,27th September - 1st October

8. $\quad$ Macdonald, M., McKay, R., Vasile, M., and Frescheville, F. B. d. "Extension of the Sun-Synchronous Orbit," Journal Guidance Control and Dynamics Vol. 33, No. 6, 2010, pp. 1935

9. Ceriotti, M., and McInnes, C. R. "An Earth Pole-Sitter using Hybrid Propulsion,"AIAA/AAS Astrodynamics Specialist Conference,Toronto, Canada,2010,2nd August - 5th August 2010

10. Ceriotti, M., and McInnes, C. R. "A Near Term Pole-Sitter using Hybrid Solar Sail Propulsion,"2nd International Symposium on Solar Sailing,New York, USA,2010,July 20-22 2010

11. Ceriotti, M., and McInnes, C. R. "Hybrid Solar Sail and SEP Propulsion for Novel Earth Observation Missions.,"61st International Astronautical Congress,Prague, CZ,2010,27 September - 1 October 2010

12. Ceriotti, M., and McInnes, C. R. "Generation of Optimal Trajectories for Earth Pole-Sitters," Journal Guidance Control and Dynamics Vol. 34, No. 3, 2011, pp. 847

13. Bate, R. R., Mueller, D. D., and White, J. E., "Fundamentals of Astrodynamics", ed., Vol., Dover Publications, Inc, 1971. p. 419-421.

14. Fortescue, P., Stark, J., and Swinerd, G., "Spacecraft Systems Engineering”, Third, ed. Wiley, Vol., 2003. p. 94.

15. Vallado, D. A., "Fundamentals of Astrodynamics and Applications", Third, ed. Press, M., Vol., 2001. p. 633.

16. Macdonald, M., and McInnes, C. R. "Analytical Control Laws for Planet-Centered Solar Sailing," Journal of Guidance, Control and Dynamics Vol. 28, No. 5, 2005, pp. 1038September - October 2005

17. Walker, M. J. H., Ireland, B., and Owens, J. "A set of Modified Equinoctial Orbital Elements," Celestial Mechanics Vol. 36, 1985, pp. 409

18. Dormand, J. R., and Prince, P. J. "A family of embedded Runge-Kutta formulae," Journal of Computational and Applied Mathematics and Statistics Vol. 6, No. 1, 1980, pp. 191980 
19. Becerra, V. M. "PSOPT Optimal Control Solver User Manual." University of Reading, 2010.

20. Hayashi, H., Cho, M., and Kuninaka, H. "High specific Impulse Microwave Discharge Ion Engine for Interplanetary explorer.," XXIInd Int. Symposium on Discharged and Electrical Insulation in Vacuum. Matsue, 2006.

21. Brophy, J. R. "Advanced Ion Propulsion Systems for Affordable Deep-Space Missions," Acta Astronautica Vol. 52, 2003, pp. 309

22. Wertz, J. R., and Larson, W. J., "Space Mission Analysis and Design”, ed., Vol. Third Edition, Microcosm Press and Kluwer Academic Publishers, 1999. p. 333.

23. King-Hele, D., "Satellite Orbits in an Atmosphere: Theory and Applications", ed., Vol., 1987. p. 256.

24. Brophy, J. R., Kakuda, R. Y., and Polk, J. E. "Ion Propulsion System (NSTAR) DS1 Technology Validation Report."

25. Wallace, N. C. "Testing of the Qinetiq T6 Thruster in Support of the ESA BepiColombo Mercury Mission," Proceedings of the 4th International Spacecraft Propulsion Conference (ESA SP-555), 2004, 2-9 June

26. D'Accolti, G., Beltrame, G., Ferrendo, E., Brambilla, L., Contini, R., Vallini, L., Mugnuolo, R., Signorini, C., Caon, A., and Fiebrich, H. "The Solar Array Photovoltaic Assembly for the Rosetta Orbiter and Lander Spacecrafts," Sixth European Conference. Porto, Portugal, 2002.

27. Racca, G. D., Marini, A., Stagnaro, L., Van Dooren, J., Di Napoli, L., Foing, B. H., Lumb, R., Volp, J., Brinkmann, J., Grunagel, R., Estublier, D., Anflo, K., Berge, S., Bodin, P., Edfors, A., Hussain, A., Kugelberg, J., Larsson, N., Ljung, B., Meijer, L., Mortsell, A., Nordeback, T., Persson, S., and Sjoberg, F. "SMART-1 Mission Description and Development Status," Planetary and Space Science Vol. 50, No. 14-15, 2002, p. 1323

28. McInnes, C. R., and Macdonald, M. "GEOSAIL: Exploring the Geomagnetic Tail Using a Small Solar Sail," Journal of Spacecraft and Rockets Vol. 38, No. 4, 2001, pp. 622July - August 2001 